\title{
Integral Representation for the Dimensionally Renormalized Feynman Amplitude
}

\author{
M. C. Bergère and F. David \\ Physique Théorique CNRS, CEN-Saclay, F-91190 Gif-sur-Yvette, France
}

\begin{abstract}
A compact convergent integral representation for dimensionally renormalized Feynman amplitudes is explicitly constructed. The subtracted integrand is expressed as a distribution in the Schwinger $\alpha$-parametric space, and is obtained by applying upon the bare integrand a new subtraction operator $R^{\prime}$ which respects Zimmermann's forest structure.
\end{abstract}

\section{Introduction}

Dimensional renormalization [1-9], first introduced by Speer and Westwater [1] and applied in the study of gauge theories by t'Hooft and Veltman [3], has proved to be an essential tool in quantum field theory. Indeed, it preserves gauge invariance, Lorentz invariance and avoids the infrared problem which appears when subtractions are performed at zeromomenta. Another advantage of this renormalization is that the Callan-Symanzik [10] function $\beta(g)$ is then independent of the dimension of space time (apart from a trivial $(D-4) \mathrm{g}$ factor) and is also independent of the mass ratios which enter the theory.

In recent years, dimensional regularization and dimensional renormalization were established on firm ground as were other kinds of renormalization, and we refer to the literature $[7,9,11]$. According to Bogoliubov-Parasiuk-Hepp (BPH) recurrence, the usual method to calculate such an amplitude is first to renormalize the smaller divergent subgraphs by extracting their poles at $D=4$, then to introduce their finite parts into larger subgraphs and reproduce the same procedure in a recurrent way. This method becomes very difficult at high orders of perturbation, dealing with overlapping divergences, spinor, coupling derivatives and gluon propagators.

On the other hand, the existence of a compact expression which, for a given Feynman graph, gives directly the dimensionally renormalized integrand is still missing. Some authors in the study of the properties of dimensional renormalization come close to achieving this goal (for instance, the $C_{H}$ operators of Breitenlohner and Maison [7] or the $\mathscr{P} \mathscr{P}_{\lambda}$ operators of Collins [6], organized in forests of divergent subgraphs). But the successive applications of these operators, 


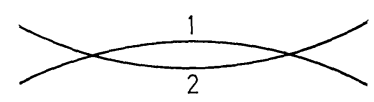

Fig. 1. The one loop graph attached to (1.1) and (1.2)

which include the implicit extraction of a pole singularity attached to a given subgraph, without giving an explicit algorithm to isolate this pole, remain to be made precise.

In this paper we solve this question and we construct explicitly a subtraction operator $R^{\prime}$, acting directly upon the bare integrand of any Feynman graph expressed in the $\alpha$-Schwinger-Symanzik representation; this operator gives the dimensionally renormalized integral as a compact, convergent integral in the $\alpha$ parametric space. The action of $R^{\prime}$ transforms the bare integrand into a distribution expressible in terms of $\theta, \delta$ distributions and their derivatives. Such distributions are shown to exist and to be integrable over the $\alpha$-parametric domain.

This paper is organized as follows: In the end of this introduction we describe, using the example of a one loop graph, the principle of the method which shall be used to obtain the dimensionally renormalized amplitude. Then, we recally the integral representation of a dimensionally regularized integrand [12]. In Sect. 2, we define the subtraction operator $R^{\prime}$; we give two examples and we comment on our result. Section 3 is devoted to the proofs and is divided into three parts: first, we show that $R^{\prime}$ defines a renormalization which satisfies the recurrence of Bogoliubov-Parasiuk [13] and we describe the corresponding counterterms; then we show by recurrence that $R^{\prime \prime}$ corresponds, subgraph by subgraph, to the extraction of the pole singularities at $D=4$ and we prove the absolute convergence of the finite parts (via the introduction of a regulator in order to avoid distributions); finally, we remove the regulator and we prove the existence and the integrability of the distributions which describe the dimensionally renormalized integrand.

We now describe the principle of the method which shall be used to obtain the dimensionally renormalized amplitude by considering the simple example of the one-loop graph of Fig. 1 which diverges logarithmically at $D=4$. For $\operatorname{Re} D<4$, the amplitude of the graph is given by the integral representation

$$
I_{G}(S, m, D)=\int_{0}^{\infty} d \alpha_{1} d \alpha_{2} \frac{\exp \left(-\left(\alpha_{1}+\alpha_{2}\right) m^{2}\right) \exp \left(-S\left(\alpha_{1} \alpha_{2}\right) /\left(\alpha_{1}+\alpha_{2}\right)\right)}{\left(\alpha_{1}+\alpha_{2}\right)^{D / 2}} .
$$

For $4<\operatorname{Re} D<6$, it has been shown in [12] that

$$
I_{G}(S, m, D)=\int_{0}^{\infty} d \alpha_{1} d \alpha_{2} \frac{\exp \left(-\left(\alpha_{1}+\alpha_{2}\right) m^{2}\right) \exp \left(-S\left(\alpha_{1} \alpha_{2}\right) /\left(\alpha_{1}+\alpha_{2}\right)\right)-1}{\left(\alpha_{1}+\alpha_{2}\right)^{D / 2}} .
$$

The function $I_{G}(S, m, D)$ behaves like $\Gamma\left(2-\frac{D}{2}\right)$ ar large imaginary $D$ and consequently a contour integral around the single pole $D=4$ can be seen as the integrals over two lines $C_{+}$and $C_{-}$as shown in Fig. 2. 


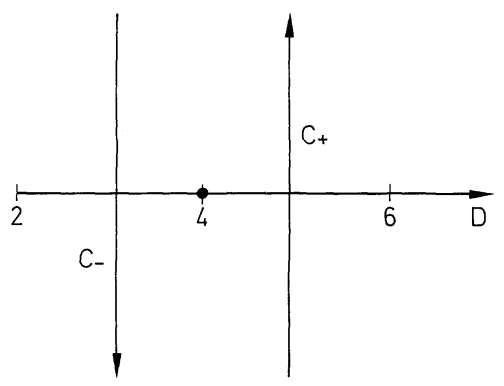

Fig. 2. Integration contour around the pole of the graph of Fig. 1

The residue of the pole is found to be

$$
r=-\int_{0}^{\infty} d \alpha_{1} d \alpha_{2} \delta\left[-\frac{1}{2} \operatorname{Ln}\left(\alpha_{1}+\alpha_{2}\right)\right]
$$

and defining the dimensionally renormalized amplitude of $G$ as

$$
I_{G}^{R^{\prime}}(S, m, D)=I_{G}(S, m, D)-\frac{r}{D-4},
$$

which is equivalent to

$$
I_{G}^{R^{\prime}}(S, m, D)=\frac{1}{2 i \pi} \oint \frac{I_{G}\left(S, m, D^{\prime}\right)}{D^{\prime}-D} d D^{\prime},
$$

where the contour in the $D^{\prime}$ complex plane encircles the two points $D^{\prime}=4$ and $D^{\prime}=D$, we obtain

$$
I_{G}^{R^{\prime}}(S, m, D)=\int_{0}^{\infty} d \alpha_{1} d \alpha_{2} \frac{\left[e^{-\left(\alpha_{1}+\alpha_{2}\right) m^{2}} e^{-\left(S \alpha_{1} \alpha_{2}\right) /\left(\alpha_{1}+\alpha_{2}\right)}-\theta\left\{-\frac{1}{2} \operatorname{Ln}\left(\alpha_{1}+\alpha_{2}\right)\right\}\right]}{\left(\alpha_{1}+\alpha_{2}\right)^{D / 2}},
$$

where $\theta(x)$ is +1 for $x>0$ and 0 for $x<0$.

The above integral, for $\operatorname{Re} D<6$, is absolutely convergent at $\alpha_{1}+\alpha_{2} \sim 0$ because of the subtraction, and at $\alpha_{1}$ or/and $\alpha_{2} \sim \infty$ because of the absence of subtraction; moreover, as wanted, the variables $m^{2}$ and $S$ are treated equally in the subtraction procedure. Another property of the above subtraction is that the zero mass limit of $I_{G}^{R^{\prime}}(S, m, D)$ exists for $2<\operatorname{Re} D<6$.

The purpose of this paper is to generalize the above example to any graph $G$ and thus to obtain in compact form a convergent integral representation for $I_{G}^{R^{\prime}}$. Several difficulties are encountered:

The above procedure of subtracting away the negative powers of $(D-4)$ in the Laurent expansion destroys unitarity as soon as multiple poles occur because it cannot be implemented by a counterterm formalism. It is necessary to suppress these negative powers by using a forest (set of non-overlapping subgraphs) subtraction formula with the condition that for each subgraph we subtract the corresponding pole at $D=4$ and nothing else. 
If we consider a graph with coupling derivatives or/and spinors, the dependence in $D$ of the $\alpha$-integrand contains a polynomial in $D$. Each power of $D$ is obtained from a contraction $g_{\mu}{ }^{\mu}$ generated by a pair of coupling derivatives or/and spinors. Now, when we calculate the residue at $D=4$ associated to a subgraph $\mathscr{S}$, one power of $D$ should be included (excluded) in the calculation if the pair of coupling derivatives or/and spinors belongs (does not belong) to $\mathscr{S}$. In other words, when we proceed to the calculation of residues for a set of subgraphs $\mathscr{S}_{1}, \ldots, \mathscr{S}_{n}$, how are we going to decide how many powers of $D$ are generated by each of the $\mathscr{S}_{i}$ 's? This problem already exists for scalar amplitudes because the subtractions over divergent subgraphs generate coupling derivatives for the corresponding reduced subgraphs. A convenient solution to these difficulties has been proposed by Ashmore [8] who introduced a multidimensional formalism. This formalism attaches a separate dimension to each subgraph and is exposed in Appendix A.

The multidimensionally regularized Euclidian Feynman amplitude is given by the integral representation

$$
I_{G}\left(p_{i}, m, D, \omega_{\mathscr{S}}\right)=\int_{0}^{\infty} \prod_{a=1}^{\ell} d \alpha_{a} Y_{G}\left(p_{i}, m, \alpha, D, \omega_{\mathscr{S}}\right),
$$

where

$$
\begin{aligned}
Y_{G}\left(p_{i}, m, \alpha, D, \omega_{\mathscr{S}}\right)= & \left\{\exp \left(-\prod_{a=1}^{\ell} \alpha_{a} m_{a}^{2}\right) S_{G}\left(p_{i}, \alpha, D, \omega_{\mathscr{S}}\right)\right. \\
& \left.\cdot \exp \left(-p d^{-1}(\alpha) p\right) P_{G}(\alpha)^{-D / 2} \prod_{\mathscr{S} \subseteq G} P_{\mathscr{S}}(\alpha)^{-\omega_{\mathscr{S}} / 2}\right\} .
\end{aligned}
$$

The functions $P_{G}(\alpha), P_{\mathscr{S}}(\alpha)$ and $p d^{-1}(\alpha) p$ are characteristic functions of the topology of the graph and of its subgraphs. The function $S_{G}\left(p_{i}, \alpha, D, \omega_{\mathcal{F}}\right)$ describes the spin and coupling derivatives part of the amplitude. In (1.7) the dimension $D$ is the dimension of space-time and the variables $\omega_{\mathscr{S}}$ are introduced according to Ashmore's formalism (Appendix A) to be the dimensions attached to every subgraph $\mathscr{S}$.

The integral (1.6) is absolutely convergent for $\left\{\operatorname{Re} D, \operatorname{Re} \omega_{\mathscr{S}}\right\}$ sufficiently small and defines by analytic continuation a meromorphic function of the variables $D$ and $\omega_{\rho g}$. As a generalization of the result obtained in [12], the analytic continuation of $I_{G}\left(p_{i}, m, D, \omega_{\mathcal{S}}\right)$ is given almost everywhere (that is away from those hyperplanes in $D$ and $\omega_{\mathscr{S}}$ where $I_{G}\left(p_{i}, m, D, \omega_{\mathscr{g}}\right)$ is singular) by the following absolutely convergent integral representation:

$$
I_{G}\left(p_{i}, m, D, \omega_{\mathscr{S}}\right)=\int_{0}^{\infty} \prod_{a=1}^{\ell} d \alpha_{a} R Y_{G}\left(p_{i}, m, \alpha, D, \omega_{\mathscr{S}}\right) .
$$

The subtraction operator $R$ is defined in [14] as

$$
R=\prod_{\mathscr{S} \subseteq G}\left(1-\tau_{\mathscr{S}}^{-2 \ell(\mathscr{\varphi})}\right)=\left[1+\sum_{\mathscr{\mathscr { S }}} \prod_{\mathscr{S} \in \mathscr{F}}\left(-\tau_{\mathscr{S}}^{-2 \ell(\mathscr{\varphi})}\right)\right],
$$

where the generalized Taylor operators $\tau_{\mathscr{S}}^{-2 \ell(\varphi)}$ are defined in [14] and where the sum over $\mathscr{F}$ runs over all forests of "divergent" subgraphs. 


\section{The Subtraction Operator $\boldsymbol{R}^{\prime}$}

We consider a graph $G$ and its Feynman amplitude $I_{G}(p, m, D)$ as defined in (1.6-8) with all $\omega_{y}$ 's $=0$. Let us consider three consecutive poles of $I_{G}: D^{-}<D^{*}<D^{+}$(if $D^{*}$ is the smallest pole of $\left.I_{G}, D^{-}=-\infty\right)$. From now on, we denote by $B^{-}$and $B^{+}$ respectively the strips $D^{-}<\operatorname{Re} D<D^{*}$ and $D^{*}<\operatorname{Re} D<D^{+}$. We intend to define the dimensionally renormalized amplitude at $D^{*}$.

We note that the operator $\tau_{\mathscr{s}}^{-2 \ell(\varphi)}$ introduced in (1.9) subtracts differently whether we stand in the strips $B^{-}$or $B^{+}$. Let us call $\tau_{\mathscr{S}}^{-}$(respectively $\tau_{\mathscr{\varphi}}^{+}$) the generalized Taylor operator relative to $\mathscr{S}$ and subtracting minimally (of degree $-2 \ell(\mathscr{S})$, where $\ell(\mathscr{S})$ is the number of internal lines in the subgraph $\mathscr{S})$ in the strip $B^{-}$(respectively $\left.B^{+}\right)$. If $\mathscr{S}$ develops no pole at $D^{*}, \tau_{\mathscr{\varphi}}^{-}=\tau_{\mathscr{\varphi}}^{+}$.

According to the requirements imposed by dimensional renormalization, namely - subtraction of the Feynman amplitude in agreement with a counterterm structure - extraction and subtraction of the singular part of the Laurent expansion around $D^{*}$ for each divergent subgraph once its interior has been subtracted - we found in the strip $D^{-}<\operatorname{Re} D<D^{+}$which contains $D^{*}$ the following convergent integral representation for the renormalized amplitude:

$$
I_{G}^{R^{\prime}}\left(p_{i}, m, D\right)=\int_{0}^{\infty} \prod_{a=1}^{\ell} d \alpha_{a} R^{\prime} Y_{G}\left(p_{i}, m, \alpha, D, \omega_{\mathscr{f}}\right) .
$$

The operator $R^{\prime}$ in (2.1) acts upon the function $Y_{G}\left(p_{i}, m, \alpha, D, \omega_{\mathscr{g}}\right)$ in the following way:

First, it subtracts the amplitude according to a forest formula of divergent subgraphs

$$
1+\sum_{\mathscr{F}} \prod_{\mathscr{S}_{\mathscr{F}}}\left(-\tau_{\mathscr{Y}}^{\prime}\right)
$$

where $\tau_{\mathscr{\varphi}}^{\prime}$ are new subtraction operators defined as

$$
\tau_{\mathscr{g}}^{\prime} F\left(\alpha, \omega_{\mathscr{g}}\right)=\tau_{\mathscr{\rho}}^{-} F\left(\alpha, \omega_{\mathscr{f}}\right)+\left(\tau_{\mathscr{\rho}}^{+}-\tau_{\mathscr{\rho}}^{-}\right) F\left(\alpha, \omega_{\mathscr{S}}\right) \theta\left(x_{\mathscr{g}}\right) .
$$

The function $\theta\left(x_{f}\right)$ is the Heaviside function and is introduced in order to perform the Cauchy integration around $D^{*}$. In this subtraction procedure the $\omega_{g}$ are considered as small positive parameters which do not change the number of subtractions of $\tau^{+}$and $\tau^{-}$. $x_{\mathscr{y}}=0$.

Second, it replaces the variables $\omega_{\mathscr{f}}$ by the operators $\frac{\partial}{\partial x_{\mathscr{\rho}}}$ acting upon the $\theta$ 's at
$=0$. The latter operation performs for every $\omega_{g}$ the complex integration

$$
\frac{1}{2 \pi} \int_{\substack{\sigma-i \infty \\ \sigma>\omega}}^{\sigma+i \infty} d\left(\operatorname{Im}_{z}\right) \frac{A(z) e^{x(z-\omega)}}{z-\omega}=A\left(\omega+\frac{\partial}{\partial x}\right) \theta(x)
$$

and generalizes to every subgraph the Cauchy extraction of the pole at $D^{*}$ performed in the example of Sect. 1.

Once the operator (2.2) is applied on $Y_{G}$, the dependence of the integrand in any $\omega_{\mathcal{S}}$ appears in terms of the form:

$$
N\left(\alpha, \omega_{\mathscr{f}}\right) Q(\alpha)^{-\omega_{\mathscr{f}} / 2},
$$




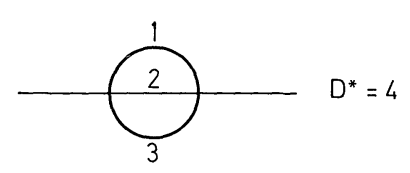

Fig. 3. The two loops quadratic diagram of Example 1

where $N$ is a polynomial of $\omega_{y}$ and $Q$ some polynomial of the $\alpha$ 's. So the second part above transforms (2.5) into either

$$
\left.N\left(\alpha, \frac{\partial}{\partial x_{\mathscr{S}}}\right) Q(\alpha)^{-1 / 2 \frac{\partial}{\partial x_{\mathscr{S}}}} \theta\left(x_{\mathscr{C}}\right)\right|_{x_{\mathscr{S}}=0}=N\left(\alpha, \frac{\partial}{\partial x_{\mathscr{S}}}\right) \theta\left[-\frac{1}{2} \log Q(\alpha)+x_{\mathscr{S}}\right]_{x_{\mathscr{S}}=0},
$$

which generates for every subgraph $\theta, \delta, \delta^{\prime}, \ldots$ distributions in the $\alpha$-space, or

$$
N\left(\alpha, \frac{\partial}{\partial x_{\mathscr{P}}}\right) Q(\alpha)^{1 / 2 \frac{\partial}{\hat{c} x_{\mathscr{S}}}} \mathbb{1}=N(\alpha, 0)
$$

if there is no $\theta$ function.

To sum up the action of the operator $R^{\prime}$, we shall write

$$
R^{\prime}=\left[1+\sum_{\mathscr{F}} \prod_{\mathscr{S} \in \mathscr{F}}\left(-\tau_{\mathscr{S}}^{\prime}\right)\right]_{\Delta}
$$

where $\Delta$ means the operation $\left.\omega_{\mathscr{S}} \rightarrow \frac{\partial}{\partial x_{\mathscr{S}}}\right|_{x_{\mathscr{S}}=0}$ once all operators $\tau_{\mathscr{S}}^{\prime}$ have been applied.

We now illustrate the rules given above by two examples:

Example 1. We consider the two loop quadratic diagram of Fig. 3 at $D^{*}=4$.

$$
Y_{G}\left(\omega_{\mathscr{S}}\right)=\frac{\exp \left(-\left(\alpha_{1}+\alpha_{2}+\alpha_{3}\right) m^{2}-p^{2} \cdot \frac{\alpha_{1} \alpha_{2} \alpha_{3}}{\alpha_{1} \alpha_{2}+\alpha_{2} \alpha_{3}+\alpha_{3} \alpha_{1}}\right)}{\left(\alpha_{1} \alpha_{2}+\alpha_{2} \alpha_{3}+\alpha_{3} \alpha_{1}\right)^{\left(D+\omega_{G}\right) / 2}\left(\alpha_{1}+\alpha_{2}\right)^{\omega_{12 / 2}}\left(\alpha_{2}+\alpha_{3}\right)^{\omega_{23} / 2}\left(\alpha_{3}+\alpha_{1}\right)^{\omega_{31 / 2}}} .
$$

The divergent subgraphs at $\left\{\begin{array}{l}D^{*}=4 \\ \omega_{\mathscr{S}}=0\end{array}\right\}$ are: $\{123\}$ quadratically divergent; $\{12\}$, $\{23\},\{31\}$ logarithmically divergent

$R^{\prime} Y_{G}\left(\omega_{\mathscr{Y}}\right)$

$$
\begin{gathered}
=Y_{G}(0)-\frac{1-\left[\left(\alpha_{1}+\alpha_{2}+\alpha_{3}\right) m^{2}+p^{2} \frac{\alpha_{1} \alpha_{2} \alpha_{3}}{\alpha_{1} \alpha_{2}+\alpha_{2} \alpha_{3}+\alpha_{3} \alpha_{1}}\right] \theta\left\{-\frac{1}{2} \operatorname{Ln}\left(\alpha_{1} \alpha_{2}+\alpha_{2} \alpha_{3}+\alpha_{3} \alpha_{1}\right)\right\}}{\left(\alpha_{1} \alpha_{2}+\alpha_{2} \alpha_{3}+\alpha_{3} \alpha_{1}\right)^{D / 2}} \\
-\frac{e^{-\alpha_{1} m^{2}} \theta\left\{-\frac{1}{2} \operatorname{Ln}\left(\alpha_{2}+\alpha_{3}\right)\right\}}{\alpha_{1}^{D / 2}\left(\alpha_{2}+\alpha_{3}\right)^{D / 2}}+\text { circ. perm. } \\
+\frac{\theta\left\{-\frac{1}{2} \operatorname{Ln}\left(\alpha_{2}+\alpha_{3}\right)\right\}\left[1-\alpha_{1} m^{2} \theta\left\{-\frac{1}{2} \operatorname{Ln}\left[\alpha_{1}\left(\alpha_{2}+\alpha_{3}\right)\right]\right\}\right]}{\alpha_{1}^{D / 2}\left(\alpha_{2}+\alpha_{3}\right)^{D / 2}}+\text { circ. perm. }
\end{gathered}
$$


Fig. 4. The two loops diagram of Example 2

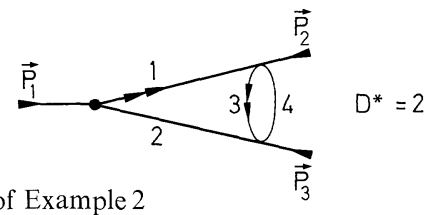

The above result differs from the usual $R$ operation [14] (subtraction at zero external momentum) first by the presence of $\theta$ distribution, second by the fact that the mass $m^{2}$ terms are subtracted, the consequence of which is that the subgraphs $\{12\},\{23\}$ and $\{31\}$ give non-zero subtraction terms although they are not generalized vertices.

Example 2 (Fig.4). This example illustrates the difficulties incoming from coupling derivatives. We concentrate on the forest of the two logarithmically divergent subgraphs at $D^{*}=2, G=\{1,2,3,4\}$ and $\mathscr{S}=\{3,4\}$.

$$
\begin{gathered}
Y_{G}\left(\omega_{\mathcal{G}}\right)=\frac{\partial}{\partial z_{1}^{2}} \frac{\partial}{\partial z_{3}^{2}}\left[P(\alpha)^{-\left(D+\omega_{G}\right) / 2}\left(\alpha_{3}+\alpha_{4}\right)^{-\omega_{\mathscr{S}} / 2}\right. \\
\left.\cdot \exp \left\{-\sum_{a=1}^{4} \alpha_{a} \bar{m}_{a}^{2}-\bar{p}_{1}^{2} \frac{\alpha_{1} \alpha_{2}\left(\alpha_{3}+\alpha_{4}\right)}{P(\alpha)}-\bar{p}_{2}^{2} \frac{\alpha_{1} \alpha_{3} \alpha_{4}}{P(\alpha)}-\bar{p}_{3}^{2} \frac{\alpha_{2} \alpha_{3} \alpha_{4}}{P(\alpha)}\right\}\right]_{z_{1}=z_{3}=0},
\end{gathered}
$$

where

$$
\begin{gathered}
\bar{m}_{a}^{2}=m_{a}^{2}-\frac{z_{a}^{2}}{4 \alpha_{a}^{2}} \quad a=1,2,3,4 \\
\bar{p}_{1}=p_{1}+\frac{z_{1}}{2 \alpha_{1}}+\frac{z_{2}}{2 \alpha_{2}} \\
\bar{p}_{2}=p_{2}-\frac{z_{1}}{2 \alpha_{1}}+\frac{z_{3}}{2 \alpha_{3}}-\frac{z_{4}}{2 \alpha_{4}} \\
\bar{p}_{3}=p_{3}-\frac{z_{2}}{2 \alpha_{2}}-\frac{z_{3}}{2 \alpha_{3}}+\frac{z_{4}}{2 \alpha_{4}} \\
P(\alpha)=\left(\alpha_{1}+\alpha_{2}\right)\left(\alpha_{3}+\alpha_{4}\right)+\alpha_{3} \alpha_{4} .
\end{gathered}
$$

In (2.12), the momentum $p_{i} \in \mathbb{R}^{D}$, the vectors $z_{1}$ and $z_{2} \in \mathbb{R}^{D} \oplus \mathbb{R}^{\omega_{G}}$, and $z_{3}$ and $z_{4} \in \mathbb{R}^{D} \oplus \mathbb{R}^{\omega_{G}} \oplus \mathbb{R}^{\omega \mathscr{S}}$.

The derivatives $\frac{\partial}{\partial z_{1}^{2}}$ and $\frac{\partial}{\partial z_{3}^{2}}$ generate the following polynomial of $\omega$ :

$$
\begin{gathered}
\frac{D_{1} D_{3}}{4} \frac{\left(\alpha_{3}+\alpha_{4}\right)\left(\alpha_{1}+\alpha_{2}+\alpha_{4}\right)}{P^{2}(\alpha)}+\frac{D_{3} \alpha_{4}^{2}}{2 P^{2}(\alpha)}+\frac{D_{3} A^{2}\left(\alpha_{1}+\alpha_{2}+\alpha_{4}\right)}{2 P(\alpha)} \\
+\frac{D_{1} B^{2}\left(\alpha_{3}+\alpha_{4}\right)}{2 P(\alpha)}+\frac{2 A \cdot B \alpha_{4}}{P(\alpha)}+A^{2} B^{2},
\end{gathered}
$$


with

$$
\begin{gathered}
A^{\mu}=\frac{-p_{1}^{\mu} \alpha_{2}\left(\alpha_{3}+\alpha_{4}\right)+p_{2}^{\mu} \alpha_{3} \alpha_{4}}{P(\alpha)}, \\
B^{\mu}=\frac{-p_{2}^{\mu} \alpha_{1} \alpha_{4}+p_{3}^{\mu} \alpha_{2} \alpha_{4}}{P(\alpha)} \\
D_{1}=D+\omega_{G}, \\
D_{3}=D+\omega_{G}+\omega_{g} .
\end{gathered}
$$

Then,

$$
\tau_{G}^{+} \tau_{\mathscr{S}}^{+} Y_{G}\left(\omega_{\mathscr{S}}\right)=\frac{D_{1} D_{3}}{4}\left(\alpha_{1}+\alpha_{2}\right)^{-\left(\frac{D+\omega_{G}+2}{2}\right)}\left(\alpha_{3}+\alpha_{4}\right)^{-\left(\frac{D+\omega_{G}+\omega_{\mathscr{P}}+2}{2}\right)}
$$

and finally

$$
\begin{gathered}
{\left[\tau_{G}^{\prime} \tau_{\mathscr{S}}^{\prime}\right]_{\Delta} Y_{G}\left(\omega_{\mathscr{S}}\right)=\left[\left(\alpha_{1}+\alpha_{2}\right)\left(\alpha_{3}+\alpha_{4}\right)\right]^{-\left(\frac{D+2}{2}\right)}} \\
\left\{\frac{D^{2}}{4} \theta_{G_{\mathscr{F}}} \theta_{\mathscr{S}}+\frac{D}{2} \delta_{G_{\mathscr{T}}} \theta_{\mathscr{S}}+\frac{D}{4} \theta_{G_{\mathscr{F}}} \delta_{\mathscr{S}}+\frac{1}{4} \delta_{G_{\mathscr{T}}}^{\prime} \theta_{\mathscr{S}}+\frac{1}{4} \delta_{G_{\mathscr{H}}} \delta_{\mathscr{S}}\right\},
\end{gathered}
$$

where the derivatives $\theta_{G_{\mathscr{F}}}^{(n)}$ means $\theta^{(n)}\left[-\frac{1}{2} \operatorname{Ln}\left\{\left(\alpha_{1}+\alpha_{2}\right)\left(\alpha_{3}+\alpha_{4}\right)\right\}\right]$ and $\theta_{\mathscr{S}}^{(n)}$ means $\theta^{(n)}\left[-\frac{1}{2} \operatorname{Ln}\left(\alpha_{3}+\alpha_{4}\right)\right]$.

To close this section, let us comment on our result. The dimensionally renormalized integrand is a distribution which is a sum of products of derivatives of the $\theta$ distribution. This fact raises a problem of existence of these products; it is shown in Sect. 3 and Appendix $C$ that the manifolds in $\alpha$ which are the support of the $\delta^{(n)}$ distributions $(n=0,1,2, \ldots)$ are neither tangent between themselves, nor tangent to the edges of the integration domain in $\alpha$. To prove the integrability of these distributions, in Sect. 3 we introduce a regulator $a>0$ and we define the functions $\theta_{a}^{(n)}$ which tend toward $\theta^{(n)}$ when $a \rightarrow 0$. Then, we prove the absolute convergence of the integrals at $a>0$ and finally we introduce test functions to show that this limit is the result of integrating the integrand at $a=0$ in the sense of distribution.

In the strip $D^{-}<\operatorname{Re} D<D^{*}$, the integral representation (1.8) for the regularized function $I_{G}(p, m, D)$ develops singularities at $D=D^{*}$, because of divergences when some $\alpha_{s}^{\prime} \rightarrow 0$. On the other hand, in the strip $D^{*}<\operatorname{Re} D<D^{+}$, the subtraction operator $R$ in (1.8) in such that no divergences appear at $D^{*}$ when some $\alpha_{s}^{\prime} \rightarrow 0$, but the divergences appear when some $\alpha_{s}^{\prime} \rightarrow \infty$ because the $R$ operator also subtracts the mass term. The $R^{\prime}$ operator in (2.8) generates for each forest a product of $\theta$ distributions (amongst other distributions) which organize themselves in such a way that subtractions are present when $\alpha_{s}^{\prime} \rightarrow 0$ and absent when $\alpha_{s}^{\prime} \rightarrow \infty$, so that the amplitude remains finite at $D=D^{*}$.

When derivative couplings or spinors are present or when we have nested quadratic divergences, we generate in $S_{G}\left(p_{i}, \alpha, D, \omega\right)$ a polynomial in $D$ of the type

$$
\sum\left\{f\left(p_{i}, \alpha\right) \prod\left[D+\sum_{\mathscr{S}} \omega_{\mathscr{S}}\right]\right\}
$$


this formulation tells what subgraphs are responsible for what power of $D$. The terms in $D^{q}$ (without $\omega_{s}^{\prime}$ ) generate only $\theta$ distributions. As mentioned above, these products of distributions are sufficient to make the $\alpha$-integrals convergent at $D=D^{*}$ in a way which is implementable by counterterms in a Lagrangian; this finite part would violate field equations and Ward identities and another finite renormalization has to be performed to restore them. The corresponding finite counterterms are responsible for the $\delta^{(n)}$ distributions.

It is known that dimensional renormalization depends on a mass scale; this dependence is implicit in our renormalization operator $R^{\prime}$, since in the Schwinger representation, the $\alpha_{s}^{\prime}$ have the dimension of the inverse of the square of a mass, and the subtractions are performed by functions $\theta^{(n)}\left[-\frac{1}{2} \operatorname{Ln} Q_{\mathscr{S}}(\alpha)\right]$.

In the massless case, the $R^{\prime}$ dimensionally renormalized amplitude exists at $D^{*}$ provided that the dimensionally regularized amplitude exists in a neightborhood of $D^{*}$. This is known to be the case for strictly renormalizable field theories at $D^{*}$, at non-exceptional momentum and when all masses are nul $[7,15]$.

\section{Construction of the Subtraction Operator $R^{\prime}$ and Convergence of the Renormalized Integral $I_{G}^{R^{\prime}}(D)$}

This section is devoted to the proofs of the assertions of Sect.2. As explained in Sect. 2, to separate the problems of convergence and those of the distributions in the integrand, we regularize the $\theta^{(n)}$ distributions by introducing $C^{\infty}$ functions $\theta_{a}^{(n)}$ given by (3.26). Then we define the regularized operator $R_{a}^{\prime}$ in a similar way to $R^{\prime}$ by a forest formula

$$
R_{a}^{\prime}=\left[1+\sum_{\mathscr{F}} \prod_{\mathscr{S} \in \mathscr{F}}\left(-\tau_{a_{\mathscr{S}}}^{\prime}\right)\right]_{\Delta}
$$

where the generalized Taylor operator $\tau_{a_{\mathscr{S}}}^{\prime}$ is defined from $\tau_{\mathscr{S}}^{\prime}$ by regularizing the $\theta^{(n)}$ distribution. Obviously, when $a=0$, we recover the $R^{\prime}$ operator. The Taylor operator $\tau_{a_{\mathscr{S}}}^{\prime}$ may be written

$$
\tau_{a_{\mathscr{S}}}^{\prime}=\tau_{\mathscr{S}}^{-}+U_{a_{\mathscr{S}}}=\tau_{\mathscr{S}}^{+}+V_{a_{\mathscr{S}}} .
$$

This section is then divided into three parts. In parts $A$ and $B$ we study the operator $R_{a}^{\prime}$ for $a \neq 0$. In part $A$ we prove that the operator $R_{a}^{\prime}$, acting upon a regularized integrand, divides it into a sum of terms which, after integration, will determine the counterterms, according to $\mathrm{BPH}$ recurrence. In part $B$ we prove the absolute convergence of the renormalized integrals

$$
I_{G}^{R_{a}^{\prime}}(p, m, D)=\int_{0}^{\infty} \prod d \alpha R_{a}^{\prime} Y_{G}(p, m, \alpha, D, \omega)
$$

in a neighbourhood of $D^{*}$, for any $a>0$. Simultaneously, we prove that the corresponding counterterms are given by the extraction of the poles of $I_{G}$ at $D^{*}$ via the modified Cauchy integral (3.18). Finally in part $C$ we study the limit $a \rightarrow 0$. First we prove that the integral $I_{G}^{R_{a}^{\prime}}$ tends toward a limit, which is the dimensionally renormalized integral. Then, as explained in Sect.2, the subtracted integrand 
$R^{\prime} Y_{G}=\lim _{a \rightarrow 0_{+}} R_{a}^{\prime} Y_{G}$ appears as a sum of products of distributions in $\alpha$ space. We give a sense to this object as a distribution. Simultaneously we prove that the "integral of this distribution" $I_{G}^{R^{\prime}}$ is perfectly meaningful and corresponds to the dimensionally renormalized integral.

A. The Counterterm Structure of the Operator $R_{a}^{\prime}$. In this section we use the notations of Sect. 2. The counterterm structure will be proved if in each strip $B^{-}$ and $B^{+}, R_{a}^{\prime}$ acts upon the regularized integrand $Y_{G}(p, m, \alpha, D, \omega)$ and gives the following characteristic decomposition

$$
R_{a}^{\prime} Y_{G}(p, m, \alpha, D, \omega)=\sum_{\{\mathscr{S}, \chi\}}\left[\prod_{\mathscr{S}} C_{\mathscr{S}}^{\chi^{ \pm}}(\alpha, D)\right] R^{ \pm} Y_{[G / U \mathscr{S}]_{\chi}}(p, m, \alpha, D)
$$

where the sum runs over all (eventually empty) families $\{\mathscr{S}, \chi\}$ of connected, one particle irreducible, disjoint subgraphs $\mathscr{S}$ which have a pole at $D^{*}$ and over the families $\chi$ of $\omega_{D^{*}}(\mathscr{S})$ derivatives relative to momenta on external legs of $\mathscr{S}$ and to internal masses of $\mathscr{S}\left(\omega_{D *}(\mathscr{S})\right.$ is the superficial degree of divergence of $\mathscr{S}$ at $\left.D^{*}\right)$. Here $R^{ \pm} Y_{[G / U \mathscr{S}]_{x}}$ are the dimensionally regularized integrands of the reduced graph $[G / U \mathscr{S}]_{\chi}$, and are defined respectively in the strips $B^{+}$and $B^{-} . C_{\mathscr{S}}^{\chi^{ \pm}}(\alpha, D)$ are functions of the $\alpha_{s}^{\prime}$ relative to $\mathscr{S}$, and are defined respectively in the strip $B^{+}$or $B^{-}$.

We prove this result in the strip $B^{-}$.

\section{Theorem 1.}

$$
R_{a}^{\prime} Y_{G}(p, m, \alpha, D, \omega)=\sum_{\{\mathscr{S}, \chi\}} \prod_{\mathscr{S}}\left[\bar{R}_{a}^{\prime(-)} Y_{\mathscr{S}}^{\chi}(\alpha, D, \omega)\right] R^{-} Y_{[G / U \mathscr{S}]_{\chi}}(p, m, \alpha, D),
$$

where the $\bar{R}_{a}^{\prime(-)}$ operator is given by a sum over all forests in $\mathscr{S}$ which do not contain the graph $\mathscr{S}$ itself,

$$
\bar{R}_{a}^{(-)}=-U_{a_{\mathscr{S}}}\left[1+\sum_{\mathscr{F} \ngtr \mathscr{S}} \prod_{\mathscr{S}^{\prime} \in \mathscr{F}}\left(-\tau_{a_{\mathscr{S}^{\prime}}^{\prime}}^{\prime}\right)\right]_{\Delta} .
$$

Proof. To prove this result, let us look at the difference between the two operators $R_{a}^{\prime}$ and $R^{-}$. In the proof, we shall forget the dependence on $a$, since we only look at algebraic rules. We have

$$
R^{\prime}-R^{-}=\sum_{\mathscr{F} \neq \emptyset}\left[\prod_{\mathscr{\varphi} \in \mathscr{F}}\left(-\tau_{\mathscr{S}}^{\prime}\right)-\prod_{\mathscr{S} \in \mathscr{F}}\left(-\tau_{\mathscr{S}}^{-}\right)\right]_{\Delta} .
$$

For any given forest $\mathscr{F}$, we have the following identity

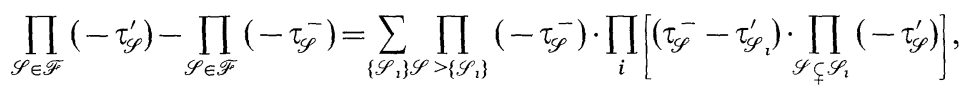

where the sum runs over all non-empty families $\left\{\mathscr{S}_{i}\right\}$ of disjoint elements of $\mathscr{F}$ (each $\mathscr{S}_{i}$ giving a pole at $\left.D^{*}\right) . \mathscr{S}>\left\{\mathscr{S}_{i}\right\}$ means that the graph $\mathscr{S}$ of $\mathscr{F}$ is either disjoint or contains some $\mathscr{S}_{i}$.

Let us now apply the operator (3.8) on the integrand $Y_{G}(D, \omega)$. (For simplicity of notation, we omit the dependence in $p$ and $m$.) In (3.8) we may take the 
dimensions $\omega_{\mathscr{g}}$ equal to zero before applying the Taylor operators, if $\mathscr{S}$ does not belong to the involved forest $\mathscr{F}$. Since $\left(\tau_{\mathscr{P}_{i}}^{-}-\tau_{\mathscr{C}_{i}}^{\prime}\right)$ is equal to $\left(-U_{\mathscr{C}_{i}}\right)$, we first apply the result of Appendix B, giving the action of the operator $\left(\tau_{\mathscr{P}_{2}}^{+}-\tau_{\mathscr{\mathscr { L }}_{2}}^{-}\right)$on $Y_{G}(D, \omega)$. We have from (B.10)

$$
\left(\tau_{\mathscr{S}_{i}}^{+}-\tau_{\mathscr{S}_{i}}^{-}\right) Y_{G}(D, \omega)=\sum_{\chi} Y_{\left[G / \mathscr{S}_{i}\right]_{\chi}}(D, \omega) Y_{\mathscr{S}_{i}}^{\chi}\left(D+\sum_{\mathscr{S}_{\mathcal{Y}} \mathscr{P}_{i}} \omega_{\mathcal{F}}, \omega\right)
$$

where the sum runs over all families of derivatives as in (3.4).

Noting by [] the operator (3.8), we obtain

$$
\begin{gathered}
{[] Y_{G}(D, \omega)=\sum_{\left\{\mathscr{S}_{i}, \chi_{i}\right\}} \prod_{\mathscr{S}_{>\left\{\mathscr{S}_{i}\right\}}\left(-\tau_{\mathscr{S}}^{-}\right)}} \\
\prod_{i} \cdot\left[\left(-U_{\mathscr{S}_{i}}\right) \cdot \prod_{\mathscr{S}_{\mathscr{C}_{1}}}\left(-\tau_{\mathscr{S}}^{\prime}\right)\right]\left\{\prod_{i} Y_{\mathscr{S}_{i}}^{\chi_{i}} \cdot Y_{\left.\left.\left[G / U \mathscr{S}_{i}\right]\right]_{\varkappa_{i}}\right\}}\right.
\end{gathered}
$$

As in $[12,16]$, any $Y_{\mathscr{P}_{2}}^{\chi_{2}}$ is a homogeneous function of $\alpha$ of such degree that it may pass through the $\tau_{\mathscr{S}}^{-}$operator by simply modifying its degree. We obtain

$$
\begin{aligned}
& {[] Y_{G}(D, \omega)=\sum_{\left\{\mathscr{S}_{1}, \chi_{i}\right\} \mathscr{S}>\left\{\mathscr{S}_{l}\right\}}\left[\left(-\tau_{\left[\mathscr{S} / U \mathscr{S}_{i}\right]}^{-}\right) Y_{\left[G / U \mathscr{P}_{1}\right]_{\chi_{i}}}(D, \omega)\right]}
\end{aligned}
$$

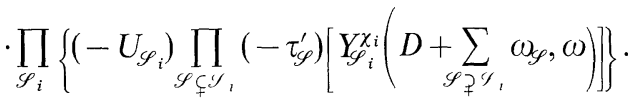

Then, to obtain the action of $R^{\prime}-R^{-}$on $Y_{G}$, we have to sum (3.11) over all nonempty forests $\mathscr{F}$ and to perform the operation $\left.\omega_{\mathscr{S}} \rightarrow \frac{\partial}{\partial x_{\mathscr{S}}}\right|_{x_{\mathscr{S}}=0}$. Reorganizing this sum as a sum over all non-empty families of disjoint divergent subgraphs and of corresponding derivatives $\{\mathscr{S}, \chi\}$, it is easy to obtain the identity (3.5). This ends the proof of Theorem 1.

We have a similar result in the strip $B^{+}$, whose proof can be performed exactly in the same way and where in (3.5-6), we change $\bar{R}_{a}^{\prime(-)}, R^{-}$and $U_{a_{\varphi}}$ respectively into $\bar{R}_{a}^{\prime(+)}, R^{+}$and $V_{a_{\mathscr{S}}}$. The operators $\bar{R}_{a}^{\prime(+)}$ and $\bar{R}_{a}^{\prime(-)}$ differ from $R_{a}^{\prime}$ only by the last operator $U_{a_{\varphi}}$ and $V_{a_{\varphi}}$ relative to the entire graph; these operators do not subtract, but on the contrary retain the divergent part at $D^{*}$ due to the graph $\mathscr{S}$.

We thus have proved the identity (3.4). The functions $C_{\mathscr{I}}^{\chi^{ \pm}}(\alpha, D)$, which are expected to give an integral representation of the counterterms in the strips $B^{+}$ and $B^{-}$respectively, are given by

$$
C_{\mathscr{S}}^{\chi^{ \pm}}(\alpha, D)=\bar{R}_{a}^{\prime \pm)} Y_{\mathscr{S}}^{\chi}(\alpha, D, \omega)
$$

Before going to part $(B)$, we prove the following result, which will be useful in part (B).

Theorem 2. Let $G$ be a divergent graph at $D^{*}$. If we consider the right hand side of (3.4), where the sum is restricted to the families $\{\mathscr{S}, \chi\}$ (eventually empty) such that the subgraphs $\mathscr{S}$ are strictly contained in $G$, we have the following identity in both strips $B^{+}$and $B^{-}$:

$$
\begin{aligned}
& {\left[\left(1-\tau_{G}^{ \pm}\right)\left(1+\sum_{\mathscr{F} \not G} \prod_{\mathscr{S} \in \mathscr{F}}\left(-\tau_{a_{\mathscr{S}}}^{\prime}\right)\right)\right]_{\Delta} Y_{G}(D, \omega)} \\
& \quad=\sum_{\substack{\{\mathscr{S}, \chi\} \\
\mathscr{S} \neq G}}\left[\prod_{\mathscr{S}} \bar{R}_{a}^{\prime( \pm)} Y_{\mathscr{S}}^{\chi}(D, \omega)\right] R^{ \pm} Y_{[G / U \mathscr{P}]_{\chi}}(D)
\end{aligned}
$$


In the left hand side we sum over all non-empty forests $\mathscr{F}$ which do not contain the graph $G$.

The proof is similar to the proof of Theorem 1 . We apply the technique of Eq. (3.7) to $\left[1+\sum_{\mathscr{F} \neq G} \prod_{\mathscr{S} \in \mathscr{F}}\left(-\tau_{a_{\mathscr{S}}}^{\prime}\right)\right]$ so that the subgraphs $\mathscr{S}_{i}$ in (3.8) are all different from $G$. Multiplying by $\left(1-\tau_{G}^{\ddagger}\right)$ we obtain (3.13).

B. Extraction of Poles and Convergence of Subtracted Integrals. We now consider the problems of convergence. We want to prove that the subtracted integrand $R_{a}^{\prime} Y_{G}(\alpha, D, \omega)$ is absolutely integrable for $D^{-}<\operatorname{Re} D<D^{+}$and corresponds to the extraction of the poles at $D^{*}$ via $\mathrm{BPH}$ recurrence. Let us recall that, if the corresponding counterterms $C_{a_{\mathscr{S}}}^{\chi}(D)$ are known for any divergent subgraph $\mathscr{S}$ in $G$, the counterterm of the graph $G$ itself is given by extracting the singular part at $D^{*}$ of the function $A_{a_{G}}$, which is defined by

$$
A_{a_{G}}(p, m, D)=I_{G}(p, m, D)+\sum_{\substack{\{\mathscr{P}, \chi\} \\ \mathscr{S} \neq G}} \prod_{\mathscr{S}} C_{a_{\mathscr{S}}}^{\chi}(D) \cdot I_{(G / U \mathscr{Y})_{\chi}}(p, m, D),
$$

where the sum runs over all non-empty families of divergent subgraphs $\mathscr{S}$ of $G$ different from $G$, as in Theorem 2 .

We shall prove that the counterterms $C_{a_{S}}^{\chi}(D)$ are meromorphic functions of $D$, whith a pole at $D^{*}$, and are given in the strips $B^{+}$and $B^{-}$respectively by the following convergent integrals:

$$
\begin{aligned}
C_{a_{\mathscr{S}}}^{\chi}(D) & =\int_{0}^{\infty} \prod d \alpha_{a}\left\{\bar{R}_{a}^{(-)} Y_{\mathscr{S}}^{\chi}(\alpha, D)\right\} \quad \text { if } \quad D \in B^{-} \\
& =\int_{0}^{\infty} \prod d \alpha_{a}\left\{\bar{R}_{a}^{(+)} Y_{\mathscr{S}}^{\chi}(\alpha, D)\right\} \quad \text { if } \quad D \in B^{+} .
\end{aligned}
$$

This result will allow us to integrate over the $\alpha$ 's the identity (3.4) of Theorem 1 in the strips $B^{-}$and $B^{+}$. We shall then obtain the counterterm expression of the subtracted integral

$$
I_{G}^{R_{a}^{\prime}}(p, m, D)=I_{G}(p, m, D)+\sum_{\mathscr{S}, \chi)} \prod_{\mathscr{S}} C_{a_{\mathscr{S}}}^{\chi}(D) I_{\left[G / U \mathscr{S}_{] \chi}\right.}(p, m, D),
$$

where the $\operatorname{sum}(\mathscr{S}, \chi)$ contains $\mathscr{S}=G$, so that from (3.14)

$$
I_{G}^{R \prime a}(p, m, D)=A_{a_{G}}(p, m, D)+\sum_{\chi} C_{a_{G}}^{\chi}(D) I_{[G / G]_{\chi}}(p, m) .
$$

In (3.16b), we see explicitly how the pole at $D^{*}$, corresponding to the entire graph $G$, cancels.

Let us now set the following theorem:

Theorem 3. For any $a>0$, the integral

$$
I_{G}^{R^{\prime} a}(p, m, D)=\int_{0}^{\infty} \prod d \alpha R_{a}^{\prime} \cdot Y_{G}(p, m, \alpha, D, \omega)
$$

is absolutely convergent for any $D$ such that $D^{-}<\operatorname{Re} D<D^{+}$. 
The corresponding counterterms have poles at $D^{*}$ and are given in the strips $B^{-}$and $B^{+}$by the convergent integral representations (3.15a and b). Moreover, this subtraction operator corresponds to the extraction of the singular part of $A_{a_{G}}$ at $D^{*}$ (defined by 3.16 ) via the Cauchy integral

$$
I_{G}^{R_{a}^{\prime}}(p, m, D)=\oint_{c} \frac{d z}{2 i \pi} \frac{A_{a_{G}}(p, m, z) e^{a(z-D)^{2}}}{z-D},
$$

where $c$ is a complex contour containing the poles of $A_{a_{G}} / z-D$ at $z=D$ and at $z=D^{*}$ (see Fig. 5).

Proof. As explained before, the function $e^{a(z-D)^{2}}$ in (3.18) is introduced to control the convergence of the integral when $|\operatorname{Im} z| \rightarrow+\infty$. To treat in a correct way the question of absolute convergence, we have to use the $L_{1}$ norm on $\alpha$ integrals. Let us note

$$
\begin{aligned}
& \left\|I_{G}(D)\right\|_{1}=\int \prod d \alpha\left|R^{-} Y_{G}(D)\right| \quad \text { if } \quad D \in B^{-} \text {, } \\
& =\int \prod d \alpha\left|R^{+} Y_{G}(D)\right| \text { if } D \in B^{+} \text {. }
\end{aligned}
$$

Since in $B^{+}$and $B^{-}$the number of subtractions is different, $\left\|I_{G}\right\|_{1}$ is analytic in $B^{+}$ and $B^{-}$, but not defined on the line $\operatorname{Re} D=D^{*}$.

Similarly, we define $\left\|C_{a_{s f}}^{\chi}(D)\right\|_{1}$ and $\left\|I_{G}^{R_{a}^{\prime}}(p, m, D)\right\|_{1}$ in $B^{+}$and $B^{-}$from the integral representations (3.15) and (3.17) (up to now, they are not proved to be finite). We now perform the $\mathrm{BPH}$ recursion on the number of loops $L(G)$ of a graph $\mathrm{G}$ to prove the theorem. The recursion hypothesis will be the following:

a) for any graph $\mathscr{S}$ such as $L(\mathscr{S})<L(G)$, Theorem 3 is satisfied.

b) Moreover, for any $\mathscr{S}$ divergent at $D^{*}$ such as $L(\mathscr{S})<L(G)$, the function $\left\|C_{a_{4}}^{\chi}(D)\right\|_{1}$ (which is finite in $B^{+}$and $B^{-}$from hypothesis a) is polynomially bounded in $B^{+}$and $B^{-}$as $|\operatorname{Im} D| \rightarrow+\infty$ for $\operatorname{Re} D$ fixed (of course for any $a>0$ ).

If $L(G)=0$, the hypothesis is trivially satisfied, since $R_{a}^{\prime} \equiv 1$. Let us prove the recursion hypothesis in the next order. According to a), the function $A_{a_{G}}(p, m, D)$ defined by (3.14) is given by the convergent integral representation in $B^{+}$and $B^{-}$ respectively

$$
A_{a_{G}}(D)=\int_{0}^{\infty} \prod_{a} d \alpha_{a} \sum_{\substack{\{\mathscr{S}, \chi\} \\ \mathscr{S} \neq G}}\left[\prod_{\mathscr{S}} \bar{R}_{a}^{( \pm)} Y_{\mathscr{S}}^{\chi}(D, \omega)\right] R^{ \pm} Y_{[G / U \mathscr{S}]_{\chi}}(D) .
$$

Using Theorem 2, this integral representation may be written:

$$
A_{a_{G}}(D)=\int_{0}^{\infty} \prod_{a} d \alpha_{a}\left[1-\tau_{G}^{ \pm}\right]\left[1+\sum_{\mathscr{F} \not G} \prod_{\mathscr{S} \in G}\left(-\tau_{a_{\mathscr{S}}}^{\prime}\right)\right]_{\Delta} Y_{G}(D, \omega) .
$$

We now perform the Cauchy integral (3.18) in order to remove from $A_{a_{G}}$ its singular part at $D^{*}$. We have to take for $C$ a contour around the two poles at $D$ and $D^{*}$; this is always possible since $D^{-}<\operatorname{Re} D<D^{+}$.

We know, from Appendix D, that any $\left\|I_{[G / \mathcal{S}]_{x}}(D)\right\|_{1}$ is polynomially bounded as $|\operatorname{Im} D| \rightarrow+\infty$. This result and part b) of the recurrence hypothesis show that $\left\|A_{a_{G}}(p, m, D)\right\|_{1}$ is polynomially bounded in $B^{+}$and $B^{-}$as $|\operatorname{Im} D| \rightarrow+\infty(\operatorname{Re} D$ being fixed). So, $\left\|A_{a_{G}}(p, m, z)\right\|_{1}\left|e^{a(z-D)^{2}}\right|$ is exponentially decreasing as $|\operatorname{Im} z| \rightarrow+\infty$. 


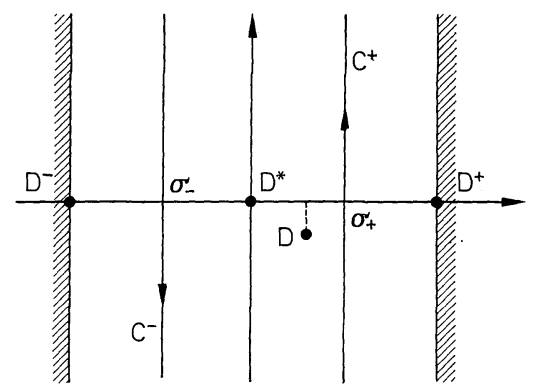

Fig. 5. Integration contour $C_{+}$and $C_{-}$, defined in (3.22-23), for the integral (3.18) in the complex $z$ plane

We may take for $C$ (see Fig. 5) the two lines $C_{+}$and $C_{-}$defined respectively by

$$
\begin{gathered}
C_{+}=\left\{z=\sigma_{+}+i y\right\} \quad \text { such that } \operatorname{Sup}\left(D^{*}, \operatorname{Re}(D)\right)<\sigma_{+}<D^{+}, \\
C_{-}=\left\{z=\sigma_{-}-i y\right\} \quad \text { such that } \quad D^{-}<\sigma_{-}<\operatorname{Inf}\left(D^{*}, \operatorname{Re} D\right) .
\end{gathered}
$$

The contour does not cross the line $\operatorname{Re} z=D^{*}$, so we may apply Fubini's theorem and invert the integrations in $z$ and in $\alpha$. Since the $\alpha$-integrand of (3.21) on $C^{+}$differs from the integrand on $C^{-}$by the subtraction operator $\tau_{G}^{+}-\tau_{G}^{-}$, we obtain:

$$
\begin{aligned}
I_{G}^{R_{a}^{\prime}}(p, m, D)= & \int_{0}^{\infty} \prod_{a} d \alpha_{a}\left\{\left[\left.\left(1-\tau_{G}^{-}\right)\right|_{z=D}-\frac{1}{2 i \pi} \int_{C_{+}} \frac{d z}{z-D} e^{a(z-D)^{2}}\left(\tau_{G}^{+}-\tau_{G}^{-}\right)\right]\right. \\
& \left.\cdot\left[1+\sum_{\mathscr{F} \neq G \in \mathscr{S} \in \mathscr{F}} \prod_{\mathscr{F}}\left(-\tau_{\mathscr{F}}^{\prime}\right)\right]_{\Delta} Y_{G}(p, m, \alpha, z, \omega)\right\} .
\end{aligned}
$$

We note that the term $\left(\tau_{G}^{+}-\tau_{G}^{-}\right)\left[1+\sum_{\mathscr{F} \neq G} \prod_{\mathscr{S}_{\mathcal{G}} \mathscr{F}}\left(-\tau_{\mathscr{F}}^{\prime}\right)\right]_{\Delta} Y_{G}(p, m, \alpha, z, \omega)$ is a function of $z$ which is a sum, relative to the forests $\mathscr{F}$, of terms of the form: $N(z) Q^{-z / 2}$, where $N(z)$ is a polynomial of $z$. The $Q$ 's are products of $P(\alpha)$ polynomials relative to reduced graphs $[\mathscr{S}]_{\mathscr{F}}$ of the forest. Using the relation

$$
\frac{1}{2 i \pi} \int_{C_{+}} \frac{d z}{z-D} \frac{e^{a(z-D)^{2}} N(z)}{Q^{z / 2}}=\left.\frac{N\left(D+\frac{\partial}{\partial x}\right)}{Q^{D / 2}} \theta_{a}\left(-\frac{1}{2} \operatorname{Ln} Q+x\right)\right|_{x=0},
$$

where $\theta_{a}$ is the convolution product of the Heaviside function with a gaussian

$$
\theta_{a}(x)=\sqrt{\frac{\pi}{a}} e^{-\frac{x^{2}}{4 a}} * \theta(x)=\sqrt{\frac{\pi}{a}} \int_{-\infty}^{+\infty} d y e^{-\frac{y^{2}}{4 a}} \cdot \theta(x-y),
$$

we deduce that the integrand in (3.24) is $R_{a}^{\prime} Y_{G}(p, m, \alpha, D, \omega)$.

From Fubini's Theorem and (3.18), we have proved that the integral (3.17) is absolutely convergent. Then, subtracting $A_{a_{G}}(D)$ from $I_{G}^{R_{a}^{\prime}}(D)$, we obtain for the counterterms relative to $G$ the convergent integral representations (3.15a) and (3.15b) in the strips $B^{+}$and $B^{-}$. Theorem 3 is proved for the graph $G$, that is part a) of the recursion hypothesis is achieved. 
To verify part b) of the recursion, let us come back to the Cauchy integral (3.18). We denote by $B_{a_{G}}^{ \pm}(p, m, \alpha, D)$ the integrand of the convergent integral representation (3.21) of $A_{a_{G}}$. The $L_{1}$ norm of $I_{G}^{R_{a}^{\prime}}$ is given, inverting $z$ and $\alpha$ integrations in (3.18), by

$$
\left\|I_{G}^{R_{a}^{\prime}}\right\|_{1}=\frac{1}{2 \pi} \int_{0}^{\infty} d \alpha\left|\int_{C} \frac{d z}{z-D} e^{a(z-D)^{2}} B_{a_{G}}^{ \pm}(p, m, \alpha, z)\right|,
$$

where $C$ is the union of the two lines $C_{+}$and $C_{-}$. Denoting by $d s$ the curvilinear absciss on $C$ (that is to say $d s=|d z|$ ), we have the inequality

$$
\left\|I_{G}^{R_{a}^{\prime}}\right\|_{1} \leqq \frac{c t e}{2 \pi} \int_{0}^{\infty} \prod d \alpha \int_{C} \frac{d s}{|z-D|} e^{-a|\operatorname{Im}(z-D)|^{2}}\left|B_{a_{G}}^{ \pm}(p, m, \alpha, z)\right| .
$$

And according to Fubini's theorem

$$
\left\|I_{G}^{R_{a}^{\prime}}\right\|_{1} \leqq \frac{c t e}{2 \pi} \int_{C} \frac{d s}{|z-D|} e^{-a|\operatorname{Im}(z-D)|^{2}}\left\|A_{a_{G}}(p, m, z)\right\|_{1} .
$$

$\left\|A_{a_{G}}\right\|_{1}$ being by hypothesis polynomially bounded as $|\operatorname{Im} z| \rightarrow \pm \infty$, the convergence of (3.29) is ensured by the $\exp \left[-a|\operatorname{Im}(z-D)|^{2}\right]$, and $\left\|I_{G}^{R_{a}^{\prime}}\right\|_{1}$ is obviously polynomially bounded as $|\operatorname{Im} D| \rightarrow \pm \infty$, as well as the counterterms relative to $G$, by (3.16b).

So we have proved the second part of the recursion hypothesis. This ends the proof of Theorem 3 .

$C$. The Limit $a \rightarrow 0_{+}$. We now look at the limit $a \rightarrow 0$ in order to recover the counterterms corresponding to the operation $R^{\prime}$. Two problems occur since in this limit the integrand appears as a sum of products of functions and distributions in $\alpha$ space:

First, the definition of such products : in Appendix C, we study the supports of the distributions $\theta^{(n)}\left[-\frac{1}{2} \operatorname{Ln} Q(\alpha)\right](n \geqq 1)$ which appear in the renormalized integrand via Eq. (3.25). Each distribution is defined only if its support is a smooth algebraic manifold. Moreover, the supports may intersect each other and/or the limits of the integration domain. Products of the corresponding distributions are defined only if these manifolds are not "tangent." These two parts are made explicit and proved in Appendix C.

Second, the integration of $R^{\prime} Y_{G}$ over the $\alpha$-space: this means that the distribution $R^{\prime} Y_{G}$ is applied over the test function 1 in the $\alpha$ space $\mathbb{R}_{+}^{\ell(G)}$. So it is not sufficient to define $R^{\prime} Y_{G}$ as a distribution over the usual spaces $\mathscr{D}\left(\mathbb{R}^{\ell}\right)$ or $\mathscr{S}\left(\mathbb{R}^{\ell}\right)$ (see [17]) to give a sense to $\int_{0}^{\infty} d \alpha R^{\prime} Y_{G^{\prime}}$.

Let us compactify $\mathbb{R}^{\ell}$ by imbedding it in the $\ell$-dimensional sphere $S_{\ell}$ via the stereographic projection and let us take for the space of test functions the space $E=C^{\infty}\left(S_{\ell}\right)$. The following theorem proves that $R^{\prime} Y_{G}$ is a distribution on the space $E$. This is sufficient for the integral $\int_{0}^{\infty} d \alpha R^{\prime} Y_{G}$ to be meaningful, since $E$ contains the function $\mathbb{1}$, and contains the usual space $\mathscr{S}\left(\mathbb{R}^{\ell}\right)$. 
Theorem 4. Given a graph $G$, for any function $\varphi$ belonging to $E$, the limit as $a \rightarrow 0_{+}$of the integral

$$
\int_{0}^{\infty} \prod_{G} d \alpha R_{a}^{\prime} Y_{G}(p, m, \alpha, D, \omega) \cdot \varphi(\alpha)
$$

exists for any $D$ belonging to the strip $D^{-}<\operatorname{Re} D<D^{+}$; and defines a distribution on $E$. In particular, the renormalized amplitude $I_{G}^{R^{\prime}}(p, m, D)$ defined by applying this distribution onto the function $\mathbb{1}$, corresponds to the usual dimensionally renormalized amplitude.

To prove the existence of the limit of (3.30), we construct recursively the integral (3.30) using a Cauchy integral generalizing (3.18), in order to control the limit $a \rightarrow 0_{+}$. Such a construction is the generalization of the construction performed in the two last sections but is rather lengthy and will not be given here; we refer to [18] for a complete proof. The existence of the limit (3.30) is sufficient to define a distribution in $E$; indeed, the space $E$ (with the usual topology induced by the sup norm on its elements and all their partial derivatives) is a countably normed space; it follows that its dual $E^{\prime}$ is complete (see [17]), and consequently the distributions $R_{a}^{\prime} Y_{G}$ converge towards a distribution in $E^{\prime}$, defined as $R^{\prime} Y_{G}$. This ends the principle of the proof.

\section{Appendix A. Multidimensional Regularization}

In this appendix we remind and adapt to our notations the construction of the dimenionally regularized Feynman integrands of [8]. Let us first recall how to obtain the Schwinger parametric form at integer dimension $N$ : Given a Feynman graph $G$ with $d_{a}$ derivative couplings $\left\{k^{\mu_{1}}, \ldots, k^{\mu_{a_{a}}}\right\}$ on each line $a(\mu$ are the Lorentz indices), each propagator is written as

$$
\begin{aligned}
\left(\prod_{i=a}^{d_{a}} k_{a}^{\mu_{2}}\right) /\left(k_{a}^{2}+m_{a}^{2}\right)= & \prod_{i}\left(-\frac{\partial}{\partial z_{a}^{\mu_{i}}}\right) \\
& \cdot\left\{\int_{0}^{\infty} d \alpha_{a} \exp \left[-\alpha_{a}\left(k_{a}^{2}+m_{a}^{2}\right)-k_{a} z_{a}\right]\right\}_{z_{a}=0},
\end{aligned}
$$

where $k_{a}$ and $z_{a}$ are $N$ dimensional vectors.

Integrating over internal momentum $k_{a}$ and taking into account momentum conservation at each vertex, we obtain the usual Schwinger representation for the Feynman integral at dimension $N$

$$
I_{G}\left(p_{i}, m_{a}\right)=\int_{0}^{\infty} \prod_{a=1}^{\ell ; G)} d \alpha_{a} \cdot Y_{G}\left(p_{i}, m_{a}, \alpha_{a}\right),
$$

where the Feynman integrand $Y_{G}$ is given by

$$
Y_{G}\left(p_{i}, m_{a}, \alpha_{a}\right)=\left\{\prod_{a=1}^{\ell i G)} \prod_{i=1}^{d_{a}}\left(-\frac{\partial}{\partial z_{a}^{\mu_{i}}}\right) Z_{G}\left(\overline{p_{i}}, \overline{m_{a}}, \alpha_{a}\right)\right\}_{z=0},
$$

and where $Z_{G}$ is given by:

$$
Z_{G}\left(\overline{p_{i}}, \overline{m_{a}}, \alpha_{a}\right)=P_{G}(\alpha)^{-N / 2} \exp \left[-\sum_{a} \alpha_{a} \overline{m_{a}^{2}}-\sum_{i, j} \bar{p}_{i} d_{G}^{-1}(\alpha)_{i j} \overline{p_{j}}\right] .
$$


In (A.3), $d_{G}(\alpha)_{i j}$ and $P_{G}(\alpha)$ are the Symanzik functions, characteristic of the topology of the graph; the dependence in the $z_{a}$ 's is reported in

$$
\begin{aligned}
& \overline{m_{a}^{2}}=m_{a}^{2}-z_{a}^{2} / 4 \alpha_{a}^{2}, \\
& \overline{p_{i}}=p_{i}+\sum_{a} \varepsilon_{i a} z_{a} / 2 \alpha_{a},
\end{aligned}
$$

where the $p_{i}$ 's are the external momenta incoming at a vertex $i$ and where $\left[\varepsilon_{i a}\right]$ is the incidence matrix of the graph $G$ and is defined as

$$
\varepsilon_{i a}=\left\{\begin{array}{l}
+1 \text { if the oriented line } a \text { points away from the vertex } i \\
-1 \text { if the oriented line } a \text { points toward the vertex } i \\
0 \text { if the line } a \text { does not contain the vertex } i
\end{array}\right.
$$

In (A.3), the dimension $N$ may become a complex dimension $D$ to define $Z_{G}$ as an analytic function of $D$ (see [12] for the definition of scalar products in dimension D).

We now define a multidimensional amplitude [8] in the following way: To each subgraph $\mathscr{S}$ we associate an additional positive dimension $\omega_{\mathscr{S}}$ so that the internal momentum $k_{a}$ belongs to the space $\mathbb{R}_{D} \underset{\mathscr{S} \supset a}{\bigoplus_{\omega_{\mathscr{S}}}} \mathbb{R}_{\text {, while the external }}$ momentum $p_{i}$ belongs to the space $\mathbb{R}_{D} \bigoplus_{\mathscr{S} \supset i} \mathbb{R}_{\omega_{\mathscr{Y}}}$. At each vertex we impose energymomentum conservation in all space $\mathbb{R}_{\omega}=\mathbb{R}_{D} \bigoplus_{\mathscr{S}} \mathbb{R}_{\omega_{\mathscr{S}}}$

$$
p_{i}=\sum_{a=1}^{\ell} \varepsilon_{i a} k_{a} \text {. }
$$

(For practical use (as in Sect.2), the external momenta $p_{i}$ may be chosen in the subspace $\mathbb{R}_{D}$.)

It is then possible to extend Schwinger representation (A.2-3) by introducing a vector $z_{a}$ in $\mathbb{R}_{D} \bigoplus_{\mathscr{S} \supset a} \mathbb{R}_{\omega_{\mathscr{S}}}$ and by computing (for $\omega_{\mathscr{S}}$ integer) the integrals

$$
\begin{aligned}
& \int \prod_{a=1}^{\ell} d^{D+} \sum_{\mathscr{S} \supset a} \omega_{\mathscr{S}} k_{a} \\
& \cdot \exp \left(-\sum_{a=1}^{\ell} \alpha_{a}\left[k_{a}^{2}+m_{a}^{2}+\frac{k_{a} \cdot z_{a}}{\alpha_{a}}\right]\right) \prod_{i=1}^{n} \delta^{D+} \sum_{\mathscr{S}{ }_{S}} \omega_{\mathscr{S P}}\left[p_{i}-\sum_{a=1}^{\ell} \varepsilon_{i a} k_{a}\right],
\end{aligned}
$$

where the scalar products in [] are taken in $\mathbb{R}_{D} \underset{\varphi(\supset a}{\mathbb{R}_{()_{y},}}$.

The above integrals may be factorized into contributions corresponding to each subspace $\mathbb{R}_{D} \oplus \mathbb{R}_{\omega_{G}}$ and $\mathbb{R}_{\omega \mathscr{\omega} \neq G}$, and (A.3) may be calculated in each of these subspaces. In each of these subspaces $\mathbb{R}_{\omega_{\mathscr{S}}}$, we integrate over the internal momenta of $\mathscr{S}$ (all internal momenta outside $\mathscr{S}$ have zero components in $\mathbb{R}_{\omega_{\mathscr{S}}}$ ). We obtain

$$
I_{G}\left(p_{i}, m_{a}, \Omega_{\mathscr{S}}\right)=\int_{0}^{\infty} \prod_{a=1}^{\ell} d \alpha_{a} Y_{G}\left(p_{i}, m_{a}, \alpha_{a}, \Omega_{\mathscr{S}}\right)
$$


with

$$
Y_{G}\left(p_{i}, m_{a}, \alpha_{a}, \Omega_{\mathscr{S}}\right)=\left.\prod_{a=1}^{\ell} \prod_{i=1}^{d_{a}}\left(\frac{-\partial}{\partial z_{a}^{\mu_{2}}}\right) \bar{Y}_{G}\left(\overline{p_{i}}, \bar{m}_{a}, \alpha_{a}, \Omega_{\mathscr{S}}\right)\right|_{z_{a}=0} .
$$

The function $\overline{Y_{G}}$ is here

$$
\bar{Y}_{G}\left(\overline{p_{i}}, \overline{m_{a}}, \alpha_{a}, \Omega_{\mathscr{S}}\right)=\left.\prod_{T \subseteq G} Z_{T}\left(\overline{p_{i}}, \overline{m_{a}}, \alpha_{a}\right)\right|_{N=\Omega_{T}},
$$

where $Z_{T}$ is defined in (A.3b); the quantities $\Omega_{\mathscr{S}}$ are $\omega_{\mathscr{S}}$ for $\mathscr{S} \neq G$ and $\Omega_{G}$ is $\left(D+\omega_{G}\right) \cdot \operatorname{In} Z_{T}$, the scalar products are taken in $\mathbb{R}_{\Omega_{T}}$ so that

$$
\begin{aligned}
& {\left[\bar{m}_{a}^{2}\right]_{G}=m_{a}^{2}-\left[z_{a}^{2}\right]_{\Omega_{G}} / 4 \alpha_{a}} \\
& {\left[\bar{m}_{a}^{2}\right]_{T}=-\left[z_{a}^{2}\right]_{\Omega_{T}} / 4 \alpha_{a} \text { for } T \neq G} \\
& {\left[\bar{p}_{i}\right]_{T}=\left[p_{i}\right]_{T}+\sum_{a} \varepsilon_{i a}\left[z_{a}\right]_{T} / 2 \alpha_{a} .}
\end{aligned}
$$

If the variables $\omega_{\mathscr{S}}$ become complex variables, the integrand in (A.8) becomes an analytic function of these variables and defines the multidimensional regularized integrand.

Let us recall that a Feynman amplitude is obtained by associating $\gamma$ Dirac and internal group matrices to (A.1) and by contracting some Lorentz and internal indices, so that the dependence of (A.8) in the $\omega_{\mathscr{S}}$ 's appears in two ways : - first, by the quantity $P_{\mathscr{S}}(\alpha)^{-\omega_{\mathscr{S}} / 2}$, - second, by various contractions between Lorentz indices which give some $g^{\mu v}, \mu$ and $v$ being relative to some $\mathbb{R}_{\omega_{\mathscr{S}}}$ and $\mathbb{R}_{\omega_{\mathscr{S}}}$. The final contraction is then,

$$
\sum_{\mu}\left[g_{\mu}^{\mu}\right]_{\mathbb{R} \omega \mathscr{S}}=\operatorname{Tr}_{\mathbb{R}_{\omega \mathscr{S}}} g=\omega_{\mathscr{S}} .
$$

(We do not emphasize the problems of the $\gamma$ matrices and of the $\gamma^{5}$ anomaly which have been extensively treated in the literature, especially in [7].)

With these algebraic rules, one defines the regularized Feynman integrand which appears to be of the form:

$$
\begin{aligned}
Y_{G}\left(p_{i}, m_{a}, \alpha_{a}, \Omega_{\mathscr{S}}\right)= & \prod_{T \subseteq G} P_{T}(\alpha)^{-\Omega_{X} / 2} \cdot S_{G}\left(p_{i}, \alpha_{a}, \Omega_{\mathscr{S}}\right) \\
& \cdot \exp \left[-\sum_{a} \alpha_{a} m_{a}^{2}-p_{i} d_{G}^{-1}(\alpha)_{i j} p_{j}\right],
\end{aligned}
$$

where $S_{G}\left(p_{i}, \alpha_{a}, \Omega_{\mathscr{S}}\right)$ is a rational function of the variables $\alpha_{a}$ which depends only polynomially on the $p_{i}$ 's and on the $\Omega_{\mathscr{S}}$.

Let us consider the convergence of the integral (A.8a). The following theorem is proved in [8].

Theorem. The integral (A.8a) is absolutely convergent for $\operatorname{Re}\left(\Omega_{\mathscr{S}}\right)$ sufficiently small, and defines by analytic continuation a meromorphic function of the variables $\Omega_{\mathscr{S}}$.

We now apply to this object the results of [12], which allow us to construct an explicit convergent integral representation of the analytic continuation of (A.8a) in all variables $\Omega_{\mathscr{S}}$. 
It is easy to prove that, following the definitions of [14], the integrand $Y_{G}\left(p_{i}, m_{a}, \alpha_{a}, \Omega_{\mathscr{S}}\right)$ admits a Taylor expansion in every Hepp's sector for every $\left\{\Omega_{\mathscr{S}}\right\}$. So we can extend without difficulty the methods of [12] to obtain the following theorem:

Theorem. The integral

$$
I_{G}\left(p_{i}, m_{a}, \Omega_{\mathscr{P}}\right)=\int_{0}^{\infty} \prod_{a} d \alpha_{a}\left\{R Y_{G}\left(p_{i}, m_{a}, \alpha_{a}, \Omega_{\mathscr{P}}\right)\right\}
$$

is absolutely convergent for $\left\{\operatorname{Re}\left(\Omega_{\mathscr{S}}\right)\right\}$ not belonging to one of the hyperplanes in $\mathbb{R}^{k}$ corresponding to a singularity of $I_{G}\left(p_{i}, m_{a}, \Omega_{\mathscr{S}}\right)$. This integral defines the analytic continuation of (A.8a) almost everywhere.

$R$ is the subtraction operator defined in [14] and in Sect. 1.

\section{Appendix B. Action of Taylor Operators upon a Multidimensional Regularized Integrand}

In this appendix, we intend to extend the results of [16] and to give the expression for

$$
\tau_{\mathscr{S}}^{n} Y_{G}\left(p_{i}, m_{a}, \alpha_{a}, \Omega_{T}\right)
$$

where $\tau_{\mathscr{S}}^{n}$ is a generalized Taylor operator defined in [14] and where $Y_{G}$ is defined in (A.8.9).

To obtain $Y_{G}$ in (A.8), we performed the Gaussian integrations of the various components of the internal momenta $k_{a}$ in each subspace $\mathbb{R}_{\Omega_{T}}$. We make here this integration in two steps as in [16]: first, integrate the internal momenta of the subgraph $\mathscr{S}$ (those momenta which have non-zero components in $\mathbb{R}_{\Omega_{T}}$, that is those corresponding to the lines of $\mathscr{S} \cap T$ ) and obtain a factor

$$
Z_{T \cap \mathscr{S}}\left(\overline{p_{i}}-\sum_{a \in \frac{T}{T \cap \mathscr{S}}} \varepsilon_{i a} k_{a}, \overline{m_{a}}, \alpha_{a}, \Omega_{T}\right) \delta^{\Omega_{T}}\left[\sum_{i \in T \cap \mathscr{S}}\left(p_{i}-\sum_{a \in \frac{T}{T \cap \mathscr{S}}} \varepsilon_{i a} k_{a}\right)\right],
$$

where scalar products are taken in $\mathbb{R}_{\Omega_{T}}$ and where, in fact, we should write a product of $\delta$ distributions over each connected component of $T \cap \mathscr{S}$. In (B.2), we denote by $\overline{p_{i}}$ the components in $\mathbb{R}_{\Omega_{T}}$ of $\left[p_{i}+\sum_{a \in S \cap T} \varepsilon_{i a} z_{a} / 2 \alpha_{a}\right]$. Then, we replace $k_{a}$ for $a \in \frac{T}{T \cap S}$ by $v\left(-\frac{\partial}{\partial z_{a}}\right)$ and we integrate over the remaining momenta of $T$. The result of this procedure is that, for any subgraph $\mathscr{S}$, we have

$$
\begin{aligned}
& \bar{Y}_{G}\left(\overline{p_{i}}, \overline{m_{a}}, \alpha_{a}, \Omega_{T}\right) \\
& \left.\quad=\sum_{T \subseteq G} Z_{T \cap \mathscr{S}}\left[\overline{p_{i}}+\sum_{a \in \frac{T}{T \cap \mathscr{S}}} \varepsilon_{i a} \frac{\partial}{\partial z_{a}}, \overline{m_{a}}, \alpha_{a}, \Omega_{T}\right)\right] Z_{\frac{T}{T \cap \mathscr{S}}}\left(\overline{p_{i}}, \overline{m_{a}}, \alpha_{a}, \Omega_{T}\right) .
\end{aligned}
$$


We may now group all functions $Z_{T \cap \mathscr{S}}$ with the same subgraph $(T \cap \mathscr{S})$ on one side, and all functions $Z_{T / T \cap \mathscr{S}}$ with the same subgraph $\left(\frac{T}{T \cap \mathscr{S}}\right)$ on the other side. Now if we take the product over all possible subgraphs $T$, we obtain

$$
\bar{Y}_{G}\left(\bar{p}_{i}, \bar{m}_{a}, \alpha_{a}, \Omega_{T}\right)=\bar{Y}_{\mathscr{S}}\left(\bar{p}_{i}+\sum_{a \in \frac{G}{\mathscr{S}}} \varepsilon_{i a} \frac{\partial}{\partial z_{a}}, \overline{m_{a}}, \alpha_{a}, \Omega_{T}^{\prime}\right) \bar{Y}_{G / \mathscr{S}}\left(\overline{p_{i}}, \overline{m_{a}}, \alpha_{a}, \Omega_{T}^{\prime \prime}\right)
$$

where

$$
\begin{aligned}
& \Omega_{T}^{\prime}=\sum_{\substack{V \text { such as } \\
V \cap \mathscr{S}=T}} \Omega_{V}, \\
& \Omega_{T}^{\prime \prime}=\sum_{\substack{V \text { such as } \\
V / \mathscr{S}=T}} \Omega_{V} .
\end{aligned}
$$

Now, $\overline{Y_{G}}$ is factorized into one function of $\alpha_{a}$ for $a \in \mathscr{S}$ and one function of $\alpha_{a}$ for $a \in \frac{G}{\mathscr{S}}$. It is then possible to apply the operator $\tau_{\mathscr{S}}^{n}$; in Sects. 2 and 3, all dimensions $\omega_{V}$ are small and positive in such a way that the number of subtractions generated by $\tau_{\mathscr{S}}^{n}$ depends only on $n$ and on the dimension $D$.

First, let us take care of the coupling derivatives on the graph $G$ by applying the derivatives $\left(-\frac{\partial}{\partial z}\right)$ in each subspace $\mathbb{R}_{\Omega_{T}}$. We use for any subgraph $\Sigma$, the property

$$
\left.\prod_{a \in \Sigma}\left(-\frac{\partial}{\partial z_{a}}\right) Z_{\Sigma}\left[\overline{p_{i}}, \overline{m_{a}}, \alpha_{a}, \Omega\right]\right|_{z=0}=S_{\Sigma}\left(p_{i}, \alpha_{a}, \Omega\right) Z_{\Sigma}\left[p_{i}, m_{a}, \alpha_{a}, \Omega\right],
$$

where $S_{\Sigma}$ is a polynomial in $p_{i}$ and $\Omega$; each monomial of $S_{\Sigma}$ satisfies the homogeneity relation

$$
h(p)-2 h(\alpha)=h,
$$

where $h(p)$ and $h(\alpha)$ are respectively the degree of homogeneity in the external momentum $p_{i}$ and in all the variables $\alpha$, and $h$ is the number of coupling derivatives.

To apply the operator $\tau_{\Sigma}^{n}$ over $S_{\Sigma} Z_{\Sigma}$, we dilate by $\varrho^{2}$ all $\alpha_{S}^{\prime}$ corresponding to the lines of $\Sigma$, and we apply $\tau_{\varrho}^{n}$; since $S_{\Sigma}$ and $P_{\Sigma}$ in $Z_{\Sigma}$ are homogeneous in $\varrho^{2}$, we have to apply $T_{\varrho}^{n-2 h(a)+L D^{*}}$ over

$$
\exp \left\{-\left[\sum_{a} \alpha_{a} m_{a}^{2}-\sum p_{i} d_{i j}^{-1} p_{j}\right] \varrho^{2}\right\}
$$

where $m_{a}$ and $p_{i}$ are the internal masses and the external momenta of the graph $\Sigma$. A Taylor expansion in $\varrho^{2}$ of this exponential is also a Taylor expansion in $m_{a}$ and $p_{i}$. Taking into account the polynomials of $p_{i}$ in $S_{\Sigma}$ we may write

$$
\tau_{\Sigma}^{n}\left\{S_{\Sigma} Z_{\Sigma}\right\}=\sum_{k=0}^{n+2 \ell<\Sigma)+\omega(\Sigma)} \frac{1}{k !} \chi_{k} \cdot\left[\frac{\partial}{\partial \chi_{k}}\left\{S_{\Sigma} Z_{\Sigma}\right\}\right]_{p_{i}=m_{a}=0}
$$


where $\ell(\Sigma)$ is the number of lines of $\Sigma, \omega(\Sigma)$ is the superficial degree of divergence of $\Sigma$ at $D=D^{*}, \chi_{k}$ is a subset of $k$ internal masses and external momenta of $\Sigma$ and $\frac{\partial}{\partial \chi_{k}}$ is the $k^{\text {th }}$ derivative in regards to the variables in $\chi_{k}$; in (B.8) summation over all $\chi_{s}^{\prime}$ is understood (as well as summation over Lorentz indices).

It is clear that the above homogeneity properties also hold over all functions $Z_{T}$ for $T \subset \Sigma$ and with $\Omega_{T}^{\prime}$ small enough. We denote by

$$
Y_{\mathscr{S}}^{\chi_{k}}\left(\alpha, \Omega_{T}^{\prime}\right)=\left.\frac{1}{k !} \frac{\partial}{\partial \chi_{k}} Y_{\mathscr{S}}\left(p_{i}, m_{a}, \alpha, \Omega_{T}^{\prime}\right)\right|_{p_{i}=m_{a}=0}
$$

and by $[G / \mathscr{S}]_{\chi_{k}}$ the reduced graph obtained by shrinking into a point the subgraph $\mathscr{S}$ and by attaching to the reduced vertex the masses of $\chi_{k}$ and the momenta of $\chi_{k}$ which are external momenta $p_{i}$ of $\mathscr{S}$ and internal coupling derivatives of $[G / \mathscr{S}]$ generated by $\varepsilon_{i a} \frac{\partial}{\partial z_{a}}$ in (B.4).

The final result used in (3.9) can be read

$$
\tau_{\mathscr{S}}^{n} Y_{G}\left(p_{i}, m_{a}, \alpha_{a}, \Omega_{T}\right)=\sum_{k=0}^{n+2 \ell(\mathscr{S})+\omega(\mathscr{S})} Y_{\mathscr{S}}^{\chi k}\left(\alpha, \Omega_{T}^{\prime}\right) Y_{[G / \mathscr{S}]_{\chi_{k}}}\left(p_{i}, m_{a}, \alpha, \Omega_{T}^{\prime \prime}\right) .
$$

\section{Appendix C}

We study here the properties of the distribution $\theta^{(n)}$ which appears in the dimensionally renormalized integrands. For simplicity we shall use a vectorial notation in $\alpha$ space : an element $\alpha=\left\{\alpha_{a}, a=1, \ell\right\}$ in $\mathbb{R}_{+}^{\ell}$ shall be noted $\alpha$. If $f(\alpha)$ is a differentiable function of the $\alpha$ 's, we shall denote the vector

$$
\left\{\frac{\partial f}{\partial \alpha_{a}}, a=1, \ell\right\} \text { by } \frac{\partial f}{\partial \alpha} \text {. }
$$

We have seen in Sect. 2 that we have to define products of distributions of the form

$$
\theta^{(n)}\left[-\frac{1}{2} \ln \left[Q_{\mathscr{S}, \mathscr{F}}(\boldsymbol{\alpha})\right]\right] \quad n \geqq 0
$$

where $\mathscr{F}$ is a forest of subgraphs of $G$ (excluding tree graphs which are never divergent), $\mathscr{S}$ a graph of $\mathscr{F}$ and where $Q_{\mathscr{S}, \mathscr{F}}$ is a polynomial in $\alpha$, defined as

$$
Q_{\mathscr{S}, \mathscr{F}}=\prod_{\substack{\mathscr{S}^{\prime} \in \mathscr{F} \\ \mathscr{S}^{\prime} \subset \mathscr{S}}} P_{\left[\mathscr{S}^{\prime}\right]_{\mathscr{F}}} .
$$

In (C.2), $P_{\left[\mathscr{S}^{\prime}\right] \mathscr{F}}$ is the Symanzik polynomial of the graph $\left[\mathscr{S}^{\prime}\right]_{\mathscr{F}}$ obtained by reducing to points in $\mathscr{S}^{\prime}$ every graph $\mathscr{S}^{\prime \prime}$ strictly contained in $\mathscr{S}^{\prime}$.

We first look at the existence of the distribution (C.1). This distribution is singular on the algebraic manifold $V_{\mathscr{S}, \mathscr{F}}$ defined by

$$
V_{\mathscr{S}, \mathscr{F}}=\left\{\boldsymbol{\alpha}: Q_{\mathscr{S}, \mathscr{F}}(\alpha)=1\right\}
$$


According to [17], this distribution is defined if, on every point of $V_{\mathscr{S}, \mathscr{F}}$ the gradient

$$
\boldsymbol{\Delta}_{\mathscr{S}, \mathscr{F}}=\frac{\partial}{\partial \boldsymbol{\alpha}} \operatorname{Ln}\left[Q_{\mathscr{S}, \mathscr{F}}(\alpha)\right]
$$

is not zero (this means that $V_{\mathscr{S}, \mathscr{F}}$ has no singular points). This is implied by the following theorem:

Theorem C. 1. On every point of $V_{\mathscr{S}, \mathscr{F}}$, the vector $\boldsymbol{\Delta}_{\mathscr{S}, \mathscr{F}}$ is different from zero.

Proof. From (C.2), $Q_{\mathscr{S}, \mathscr{F}}$ is an homogeneous polynomial of degree $L(\mathscr{S})$, where $L(\mathscr{S})$ is the number of loops of $\mathscr{S}$ (which is non-zero since $\mathscr{S}$ is not a tree graph). Applying the Euler relation to $Q$ we obtain, if $\alpha \in V_{\mathscr{S}, \mathscr{F}}$

$$
L(\mathscr{S})=\boldsymbol{\alpha} \cdot \frac{\partial}{\partial \boldsymbol{\alpha}} Q_{\mathscr{S}, \mathscr{F}}=\boldsymbol{\alpha} \cdot \boldsymbol{\Delta}_{\mathscr{S}, \mathscr{F}} .
$$

This ensures that $\boldsymbol{\Delta}_{\mathscr{S}, \mathscr{F}}$ is never null.

We now look at the product of such distributions. From Sect. 2, the integrand in $\mathbb{R}^{\ell}$ appears to be a sum of terms of the type

$$
\prod_{\mathscr{S} \in \mathscr{F}} \theta^{(n \mathscr{Y})}\left[-\frac{1}{2} \operatorname{Ln} Q_{\mathscr{S}, \mathscr{F}}(\alpha)\right] \cdot \prod_{a=1}^{\ell} \theta\left(\alpha_{a}\right),
$$

where $\mathscr{F}$ is a forest of subgraphs $\mathscr{S}(L(\mathscr{S}) \neq 0)$ and the distributions $\theta\left(\alpha_{a}\right)$ are introduced to take into account the integration over the positive $\alpha_{a}$ 's only. Such a product is defined if the various manifolds $V_{\mathscr{S}, \mathscr{F}}$ and the hyperplanes $P_{a}$ defined by

$$
P_{a}=\left\{\alpha: \alpha_{a}=0\right\}
$$

are never "tangent" at their intersections. This is ensured by the following theorem.

Theorem C. 2. Given any subforest $\mathscr{F}_{I}=\left\{\mathscr{S}_{i}, i \in I\right\}$ of the forest $\mathscr{F}$ and any subgraph $\mathscr{S}_{0}$ of $G$ (eventually empty), if the intersection

$$
V=\left(\bigcap_{i \in I} V_{\mathscr{S}_{i}, \mathscr{F}}\right) \cap\left(\bigcap_{a \in \mathscr{S}_{0}} P_{a}\right)
$$

is not empty, at every point of $V$ the vectors $\boldsymbol{\Delta}_{\mathscr{S}_{l}, \mathscr{F}}(i \in I)$ and the vectors $\mathbf{n}_{a}$ defined as

$$
\mathbf{n}_{a}=\frac{\partial}{\partial \boldsymbol{\alpha}} \alpha_{a}\left(a \in \mathscr{S}_{0}\right)
$$

are linearly independent.

Proof. We first prove the theorem when the graph $\mathscr{S}_{0}$ is empty. Then, we first note that according to (C.2), every polynomial $Q_{\mathscr{S}_{i}, \mathscr{F}}$ may be written as a product of polynomials $R_{i}$ relative to the graphs of the subforest $\mathscr{F}_{I}$ contained in $\mathscr{S}_{i}$ :

$$
Q_{\mathscr{S}_{i}, \mathscr{F}}=\prod_{j \in I: \mathscr{S}_{j} \subset \mathscr{S}_{i}} R_{j},
$$

where $R_{j}$ is defined as the product of the polynomials $P_{[\mathscr{S}]_{\mathscr{F}}}$ relative to the subgraphs $\mathscr{S}$ of $\mathscr{F}$ which are contained in $\mathscr{S}$, but not contained in any subgraph $\mathscr{S}_{k}$ of $\mathscr{F}_{I}$ strictly contained in $\mathscr{S}_{j}$. 
From (C.8) and (C.10), the point $\alpha$ belongs to $V$ if and only if, for any $i \in I$, $R_{i}(\alpha)=1$. Then, if $\alpha$ belongs to $V$, we have

$$
\boldsymbol{\Delta}_{\mathscr{S}_{i}, \mathscr{F}}(\boldsymbol{\alpha})=\sum_{\substack{j \in I \\ \mathscr{S}_{j} \subset \mathscr{S}_{\imath}}} \frac{\partial}{\partial \boldsymbol{\alpha}} R_{j}(\boldsymbol{\alpha}) .
$$

By the same homogeneity argument used in Theorem C.1, the vectors $\frac{\partial}{\partial \boldsymbol{\alpha}} R_{i}(\boldsymbol{\alpha})$ are non-zero; since they are orthogonal $\left[\right.$ that is $\frac{\partial}{\partial \boldsymbol{\alpha}} R_{i}(\boldsymbol{\alpha}) \cdot \frac{\partial}{\partial \boldsymbol{\alpha}} R_{j}(\boldsymbol{\alpha})=0$, if $\left.i \neq j\right]$, they are linearly independent. This result, with (C.11), ensures that at every point of $\bigcap_{i \in I} V_{\mathscr{S}_{i}, \mathscr{F}}$, the vectors $\boldsymbol{\Delta}_{\mathscr{S}_{i}, \mathscr{F}}(\boldsymbol{\alpha})$ are linearly independent.

We now consider the case where $\mathscr{S}_{0}$ is not empty. Since the vectors $\mathbf{n}_{a}$ are orthogonal, we may restrict ourselves to the subspace

$$
E=\bigcap_{a \in \mathscr{S}_{0}} P_{a}=\left\{\alpha: \alpha_{a}=0: a \in \mathscr{S}_{0}\right\}
$$

To any subgraph $\mathscr{S}$ of $\mathscr{F}$ we associate the subgraph

$$
\overline{\mathscr{S}}=\left[\mathscr{S} / \mathscr{S} \cap \mathscr{S}_{0}\right]
$$

of $\left[G / \mathscr{S}_{0}\right]$ and we consider the forest

$$
\overline{\mathscr{F}}=\{\overline{\mathscr{S}}: \mathscr{S} \in \mathscr{F}\} .
$$

The restriction to the subspace $E$ of the polynomials $P_{[\mathscr{G}]_{\mathscr{F}}}$ may be proved to be (see [16]):

$$
\begin{array}{ll}
P_{[\mathscr{Y}]_{\mathscr{F}}}(\alpha)=P_{[\overline{\mathscr{Y}}]_{\overline{\mathscr{F}}}} \text { if } \quad L\left([\overline{\mathscr{S}}]_{\overline{\mathscr{F}}}\right)=L\left([\mathscr{S}]_{\mathscr{F}}\right), \\
P_{[\mathscr{S}]_{\mathscr{F}}}(\alpha)=0 \quad \text { if } \quad L\left([\overline{\mathscr{S}}]_{\overline{\mathscr{F}}}\right)<L\left([\mathscr{S}]_{\mathscr{F}}\right) .
\end{array}
$$

The manifold $V$ defined by (C.8) is not empty only if for any $\mathscr{S}$ of $\mathscr{F}$ contained in any $\mathscr{S}_{i \in I}$ we have $L\left(\mathscr{S} \cap \mathscr{S}_{0}\right)=0$.

In that case, we are reduced to the problem of the independence of the vectors

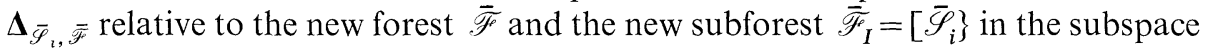
$E$. The proof follows similar to the case $\mathscr{S}_{0}$ empty. This ends the proof of Theorem C.2.

\section{Appendix D}

This appendix is devoted to the proof of various properties of the dimensionally regularized integrals as $|\operatorname{Im} D| \rightarrow+\infty$, which are used in Sect. 3.B.

Let $G$ be a Feynman graph (with internal non-zero masses). According to [12], its regularized integral has the following integral representation provided that $\operatorname{Re} D$ is different from any pole characteristic of the graph $G$.

$$
I_{G}(p, m, D)=\int_{0}^{\infty} \prod_{a=1}^{\ell} d \alpha_{a} R\left\{Y_{G}(p, m, D, \alpha)\right\} .
$$


According to the appendix $C$ of [12], Eq. (D.1) may be decomposed in absolutely convergent integrals associated to different Hepp's sectors and different equivalent classes $\Gamma$ of nests. A Hepp sector is given by an ordering of the lines

$$
s=\left\{\alpha ; \alpha_{a_{\ell}} \geqq \alpha_{a_{t-1}} \ldots \geqq \alpha_{a_{1}}\right\} .
$$

We perform the change of variables into Hepp variables, that is to say

$$
\alpha_{a_{i}}=\prod_{j=1}^{\ell} \beta_{j}
$$

Then, each contribution is of the form

$$
\begin{aligned}
& \int_{0}^{+\infty} d \beta_{\ell} \beta_{\ell}{ }^{a-\frac{D L}{2}-1} \int_{0}^{1} \prod_{i=1}^{\ell-1} d \beta_{i} \beta_{i}^{a_{i}-\frac{D L_{i}}{2}-1} \int_{0}^{1} \prod_{j \in J_{l}} d \chi_{j}^{i}\left(1-\chi_{j}^{i}\right)^{b_{j}^{i}} \\
& \cdot \exp \left(-\prod_{i=K}^{\ell} \beta_{i} \chi_{r_{\imath}-1}^{i}\left[m_{i_{K}}^{2}+E(m, p, \beta, x)\right]\right) F\left(p, m, D, \beta_{i} \prod_{j \in J_{i}} \chi_{j}^{i}\right) .
\end{aligned}
$$

The variables $\chi_{j}^{i}, j \in J_{i}\left(J_{i}\right.$ is some subset of $\left.\left\{1, \ldots, r_{i}-1\right\}\right)$, are introduced to take into account the subtractions due to divergent subgraphs associated to the class $\Gamma$. $K$ is some integer $\leqq \ell$.

The variables $\chi_{r_{l}-1}^{i}$ in $\exp \left(-\prod_{i=K}^{\ell} \beta_{i} \chi_{r_{i}-1}^{i}\right)$ are present if and only if the graph $G$ is subtracted; if not, they are set equal to 1 in (D.4).

The exponents $a_{j}(j=1, \ell)$ and $b_{j}^{i}$ are such that:

$$
\begin{gathered}
0<\operatorname{Re}\left(a_{\ell}-\frac{D L}{2}\right)<1, \quad \text { if } G \text { is subtracted, } \\
\operatorname{Re}\left(a_{\ell}-\frac{D L}{2}\right)>0, \quad \text { if } \quad G \text { is not subtracted, } \\
a_{i}-a_{\ell}+\frac{D}{2}\left(L-L_{i}\right)>0, \quad K \leqq i<\ell, \\
a_{i}-\frac{D L_{i}}{2}>0, \quad i<K, \\
b_{j}^{i} \geqq 0 .
\end{gathered}
$$

The function $E\left(p, m, \beta_{i}\right)$ is a continuous non-negative function of the $\beta_{i}$ 's $(i=1, \ell-1)$.

The function $F\left(p, m, D, \beta_{i}\right)$ is a continuous function of the $\beta_{i}$ 's $(i=1, \ell=1)$ and is polynomially bounded as $|\operatorname{Im} D| \rightarrow+\infty$.

The conditions (D.5, D.6 and D.7) were sufficient to ensure the absolute convergence of the integral (D.4). We now study the limit $|\operatorname{Im} D| \rightarrow+\infty$.

Lemma 1. For Re $D$ fixed, different from any poles of $G, I_{G}(p, m, D)$ is exponentially bounded as $|\operatorname{Im} D| \rightarrow \infty$; more precisely

$$
\forall \alpha>\frac{\pi \cdot L(G)}{4}, \quad \exists M>0:\left|I_{G}(p, m, D)\right| \leqq M e^{-\alpha|\operatorname{Im} D|} .
$$


Proof. Let us integrate (D.4) over $\beta_{\ell}$. We obtain

$$
\begin{gathered}
\Gamma\left(a_{\ell}-\frac{D L}{2}\right) \int_{0}^{1} \prod_{i<K} d \beta_{i} \beta_{i}^{a_{\ell}-\frac{D L_{i}}{2}-1} \int_{0}^{1} \prod_{i=K}^{\ell-1} d \beta_{i} \beta_{i}^{a_{\imath}-a_{\ell}+D \frac{i L-L_{2}}{2}} \int_{0}^{1} \prod_{j \in J_{\ell}} d \chi_{j}^{i} \\
\left(1-\chi_{j}^{i}\right)^{b_{j}^{i}} \prod_{i=K}^{\ell}\left(\chi_{r_{i}-1}^{i}\right)^{\frac{D L}{2}-a_{\ell}} \cdot F(p, m, D, \beta \chi)\left(m_{i_{K}}^{2}+E\right)^{\left(\frac{D L}{2}-a_{\ell}\right)} .
\end{gathered}
$$

At $\operatorname{Re} D$ fixed, away from a pole, the integrals over the $\beta_{i}$ 's and the $\chi_{j}^{i}$, s are convergent (from the conditions (D.5, D.6 and D.7). The modulus is majored by the integral of the modulus, which is polynomially bounded as $|\operatorname{Im} D| \rightarrow+\infty$. So it is easy to see that the integral is polynomially bounded.

From the asymptotic behaviour of the $\Gamma$ function:

$$
\Gamma(x+i y) \sim \sqrt{2 \pi}|y|^{x-1 / 2} e^{-\frac{\pi}{2}|y|}, \text { as }|y| \rightarrow+\infty,
$$

we deduce the result of Lemma D.1.

Lemma 2. For $\operatorname{Re} D$ fixed, different from any poles of $G$, the $L_{1}$ norm of the regularized integral $\left\|I_{G}(p, m, D)\right\|_{1}$ as defined in (3.19) is polynomially bounded as $|\operatorname{Im} D| \rightarrow+\infty$.

Proof. Since $I_{G}(p, m, D)$ is a sum of integrals of the form (D.4), its $L_{1}$ norm is majored by the sum of the $L_{1}$ norms of the integrals (D.4). Those $L_{1}$ norms are integrals of the form

$$
\begin{gathered}
\int_{0}^{+\infty} d \beta_{\ell} \beta_{\ell}^{a_{\ell}-\frac{\operatorname{Re} D \cdot L}{2}-1} \int_{0}^{1} \prod_{i=1}^{\ell-1} d \beta_{i} \beta_{i}^{a_{2}-\frac{\operatorname{Re} D \cdot L_{i}}{2}-1} \int_{0}^{1} \prod_{j \in J_{i}} d \chi_{j}^{i}\left(1-\chi_{j}^{i}\right)^{b_{J}} \\
\cdot \exp \left(-\prod_{i=K}^{\ell} \beta_{i} \chi_{r_{l}-1}^{i}\left(m_{i_{K}}^{2}+E\right)\right) \quad|F(p, m, D, \beta \chi)| \frac{\operatorname{Re} D \cdot L}{2}-a_{\ell}
\end{gathered}
$$

$|F|$ is a continuous function of $\beta \chi$, polynomially bounded as $|\operatorname{Im} D| \rightarrow+\infty$. Since $|F|$ contains the only dependence in $|\operatorname{Im} D|$ of (D.11), and since the integral is absolutely convergent, the integral (D.11) is polynomially bounded as $|\operatorname{Im} D| \rightarrow+\infty$. The result of Lemma D. 2 is then proved.

Acknowledgements. We wish to thank J. Lascoux for his interest and for his useful comments.

\section{References}

1. Speer, E.R., Westwater, M.J.: Ann. Inst. Henri Poincaré A 14, 1-55 (1971)

2. Bollini, C.G., Giambiagi, J.J.: Phys. Lett. B40, 566-568 (1972); Acta Phys. Austr. 38, 211 (1973)

3. 't Hooft, G., Veltman, M.: Nucl. Phys. B44, 189-213 (1972)

4. Speer, E.R.: J. Math. Phys. 15, 1-6 (1974)

5. de Vega, H.J., Schaposnik, F.A.: J. Math. Phys. 15, 1998-2000 (1974) 
6. Collins, J.C.: Nucl. Phys. B92, 477-506 (1975)

7. Breitenlohner, P., Maison, D.: Commun. Math. Phys. 52, 11, 39, 55-75 (1977)

8. Ashmore, J.F.: Commun. Math. Phys. 29, 177-187 (1973)

9. Speer, E.R.: Commun. Math. Phys. 37, 83-92 (1974);

Speer, E.R.: Dimensional and analytic renormalization. In: The Erice Summer School on "Renormalization Theory", Velo, G., Wightman, A.S. (eds.) (1975)

10. Callan, C.G.: Phys. Rev. D2, 1541-1547 (1970)

Symanzik, K.: Commun. Math. Phys. 18, 227-246 (1970); 23, 49-86 (1971)

11. Wilson, K.: Phys. Rev. D7, 2911-2926 (1973) Appendix

Mahoux, G.: Private communication

12. Bergère, M.C., David, F.: J. Math. Phys. 20, 1244-1255 (1979)

13. Bogoliubov, N.N., Parasiuk, G.: Acta Math. 97, 227 (1957)

14. Bergère, M.C., Zuber, J.B.: Commun. Math. Phys. 35, 113-140 (1974)

Bergère, M.C., Lam, Y.M.P.: J. Math. Phys. 17, 1546-1557 (1976)

15. David, F.: Thèse $3^{\text {ième }}$ Cycle, Mars 1978

16. Bergère, M.C., Lam, Y.M.P.: Asymptotic expansion of Feynman amplitudes, Part II, the divergent case. Freie Universität Berlin, Preprint HEP, May 74/9 (1974)

17. Gelfand, I.M., Shilow, G.E.: Generalized Functions, Vol.1 and 2. New York and London: Academic Press Inc., 1964-1968

Schwartz, L.: Theorie des distributions, Paris: Hermann, 1957-1959

18. A detailed proof of theorem 4 is available in Saclay Preprint DPh. T. 80/23, March 1980

19. In Ashmore's construction, additional dimensions are introduced for generalized (dominant) vertices only

Communicated by R. Stora

Received April 8, 1980; in revised form November 10, 1980 